\title{
An analytical model for the translaminar fracture toughness of fibre composites with stochastic quasi-fractal fracture surfaces
}

\author{
Soraia Pimenta ${ }^{1, *}$, Silvestre T. Pinho \\ Department of Aeronautics, South Kensington Campus, Imperial College London. London SW7 $2 A Z$, \\ United Kingdom
}

\begin{abstract}
The translaminar fracture toughness of fibre-reinforced composites is a size-dependent property which governs the damage tolerance and failure of these materials. This paper presents the development, implementation and validation of an original analytical model to predict the tensile translaminar (fibre-dominated) toughness of composite plies and bundles, as well as the associated size effect. The model considers, as energy dissipation mechanisms, debonding and pull-out of bundles from quasi-fractal fracture surfaces; the corresponding lengths are stochastic variables predicted by the model, based on the respective bundle strength distributions and fracture mechanics. Parametric studies show that composites are toughened by stronger fibres with large strength variability, and intermediate values of interfacial toughness and friction. Predictions are validated against four different composite ply systems tested in the literature, proving the models ability to capture not only size effects, but also the influence of different fibres and resins. Keywords: A. strengthening and mechanisms, B. fibre-reinforced composite material, C. probability and statistics, Size effects.
\end{abstract}

\section{Introduction}

The translaminar fracture toughness $(\mathcal{G})$ of UniDirectional (UD) Fibre Reinforced Polymers (FRPs) is the energy required to fracture the material perpendicularly to the fibre direction (per unit nominal area). This property governs the damage tolerance of structures with load-aligned fibres, as well as the strength of components with geometric

\footnotetext{
${ }^{*}$ Corresponding author. Fax: +44 (0)20 75941974.

Email address: soraia.pimenta@imperial.ac.uk (Soraia Pimenta)

${ }^{1}$ Present address: Department of Mechanical Engineering, South Kensington Campus, Imperial College London, SW7 2AZ, United Kingdom

Pimenta S, Pinho ST (2014). An analytical model for the translaminar fracture toughness of fibre composites with stochastic quasi-fractal fracture surfaces. Journal of the Mechanics and Physics of Solids 66, 78-102.DOI:10.1016/j.jmps.2014.02.001
} 


\begin{tabular}{|c|c|}
\hline \multicolumn{2}{|c|}{ Nomenclature } \\
\hline Uppercase variables & Lowercase greek variables \\
\hline$A \quad$ cross sectional area & $\alpha \quad$ aspect ratio \\
\hline$C \quad$ perimeter & diameter \\
\hline $\mathrm{CoV}$ coefficient of variation & stress concentrations parameter (Eq. 14) \\
\hline$E \quad$ elastic modulus & $\begin{array}{ll}\lambda & \text { stress field slope }\end{array}$ \\
\hline Cumulative Distribution Function (CDF) & $\sigma \quad$ longitudinal stress \\
\hline extreme-value CDF & shear (yield) stress \\
\hline (translaminar) fracture toughness & $\psi \quad$ toughness parameter (Eq. 14) \\
\hline length (stochastic) & \\
\hline extreme-value length (stochastic) & Superscripts \\
\hline survival probability, complementary CDF & 0 single-fibre composite \\
\hline extreme-value complementary CDF & fibre \\
\hline volume fraction & {$[i]$ bundle level } \\
\hline$W \quad$ energy dissipated in fracture & $\infty$ remote \\
\hline \multicolumn{2}{|l|}{$X \quad$ longitudinal tensile strength (stochastic) } \\
\hline & Subscripts \\
\hline Lowercase roman variables & 0 Weibull scale parameter \\
\hline$a \quad$ debonding distance & deb debonding \\
\hline$c \quad$ coordination number & $\mathrm{H}$ hexagonal arrangement \\
\hline translaminar toughness components & mode-II delamination \\
\hline bundle level & mean value \\
\hline stress concentrations factor & frictional \\
\hline length & pull-out \\
\hline Weibull shape parameter & quadrangular arrangement \\
\hline number of fibres & reference length \\
\hline interfibre spacing & SL interface, shear-lag \\
\hline thickness & uniform stress state \\
\hline
\end{tabular}

discontinuities; it is also dramatically affected by size effects (Laffan et al., 2012), which raises a challenge for the simulation of damage tolerant structures. This paper presents a model for the translaminar tensile toughness of FRPs, based on fibre and interfacial properties and assuming the formation of stochastic quasi-fractal fracture surfaces.

The translaminar toughening mechanisms of FRPs have been extensively investigated (Kim and Mai, 1991), and methods to measure the corresponding fracture toughness have been developed (Laffan et al., 2012). Composites are orders of magnitude tougher than their constituents, due to the formation of intricate 3D fracture surfaces with large interfacial debonds and pulled-out fibres and bundles (Figures 1 and 2).

Laffan et al. (2010) recently reported size effects on the translaminar toughness of FRPs, by testing cross-ply Compact Tension (CT) specimens with 0.125 or $0.250 \mathrm{~mm}$ thick $0^{\circ}$ layers. The measured translaminar toughness of the thicker layers was nearly twice the value for the thinner ones, reportedly due to much larger pull-out features (Figure 1). Subsequent finite elements simulations of open-hole specimens (Chen et al., 2013) proved that incorporating such dependence in numerical models is crucial to 


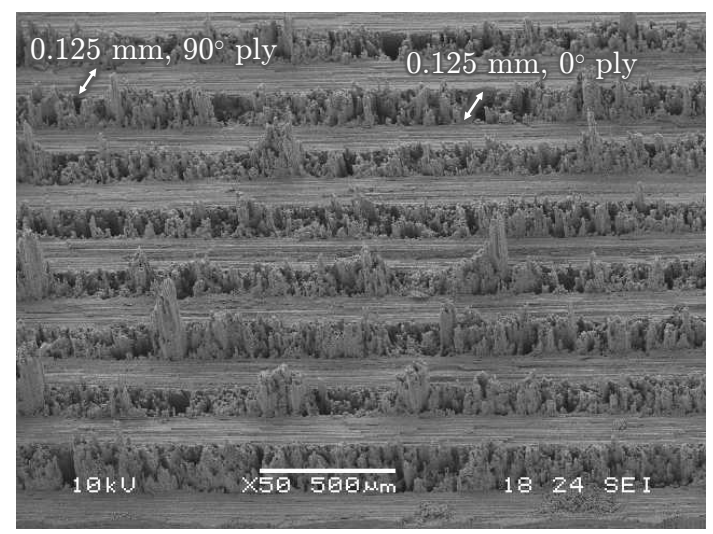

(a) Fracture surface with $0.125 \mathrm{~mm}$ thick $0^{\circ}$ layers and $\mathcal{G}=65 \mathrm{~kJ} / \mathrm{m}^{2}$.

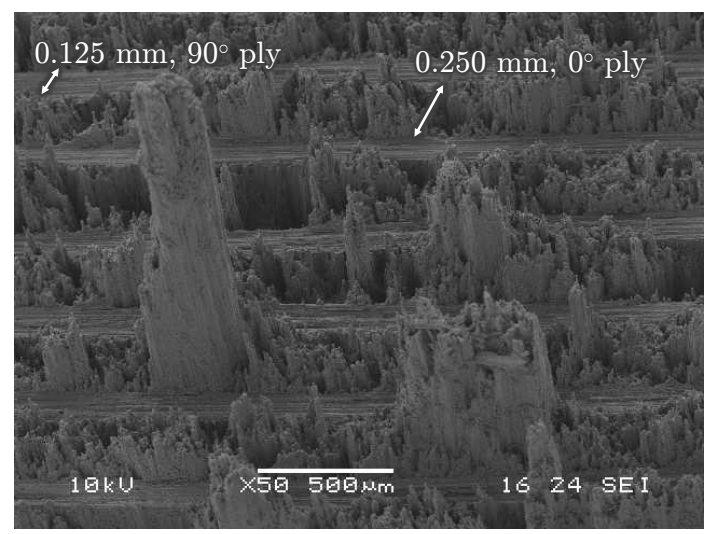

(b) Fracture surface with $0.250 \mathrm{~mm}$ thick $0^{\circ}$ layers and $\mathcal{G}=132 \mathrm{~kJ} / \mathrm{m}^{2}$.

Figure 1: Size effects on the translaminar fracture of UD carbon-epoxy plies (after Laffan et al., 2010).

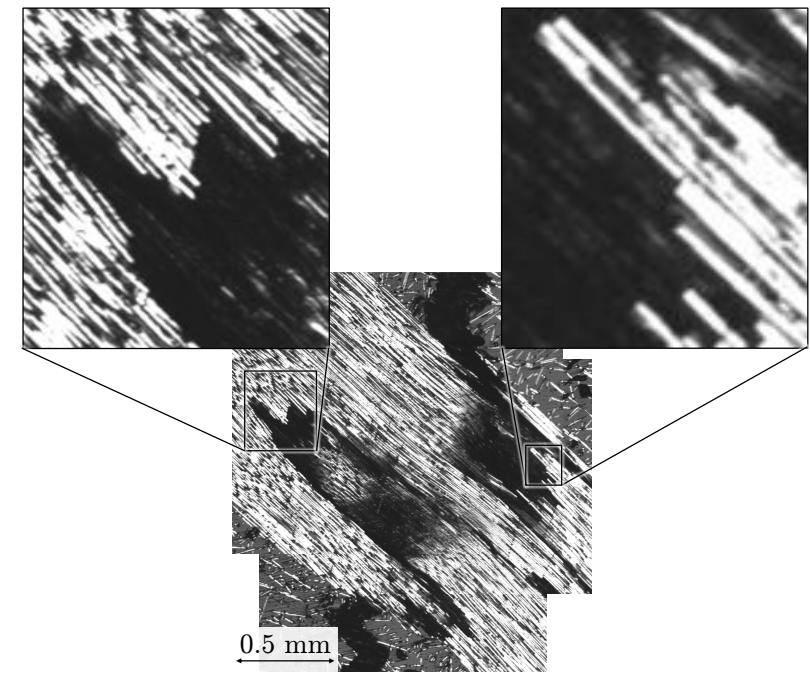

(a) Fibre bundle in a recycled composite (adapted from Pimenta et al., 2010).

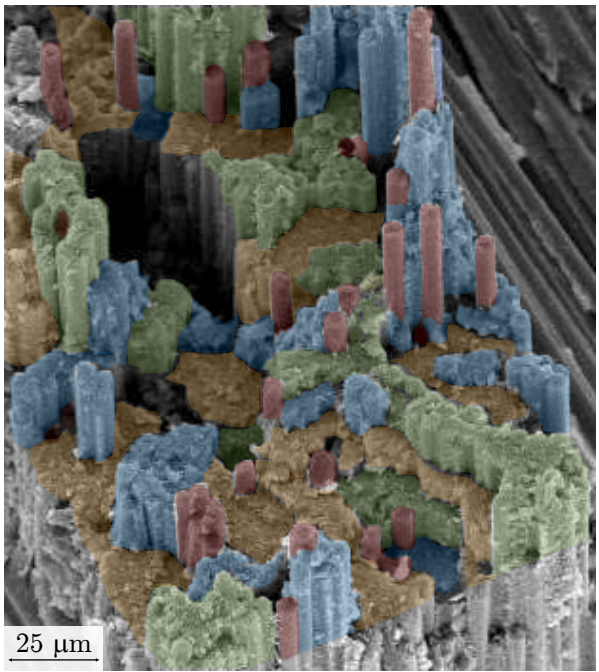

(b) UD FRP ply.

Figure 2: Hierarchical and quasi-fractal features on the translaminar fracture surface of UD composites.

replicate experimental results.

Additionally, Pimenta et al. (2010) observed that the fracture surface of recycledfibre bundles is hierarchical and statistically self-similar (see Figure 2a, with individual fibres pulled-out from the surface of small bundles, which are themselves pulled-out from larger bundles). Further analysis confirmed that these features are also characteristic of virgin UD composites (Figure 2b).

Most authors (e.g Gao et al., 1988, Kelly, 1970, Kim and Mai, 1991, Wells and Beaumont, 1985b) agree that interfacial debonding and pull-out (hereby indicated by 
the subcripts deb and po) are the main toughening mechanisms of UD composites; the corresponding contributions (in terms of energy dissipated per fibre, $W^{\mathrm{f}}$, and work of fracture of the composite, $\mathcal{R}$ ) can be approximated as (Kim and Mai, 1991):

$$
\left\{\begin{array}{lll}
W_{\mathrm{deb}}^{\mathrm{f}}=C^{\mathrm{f}} \cdot \mathcal{G}_{\mathrm{SL}} \cdot l_{\mathrm{deb}} & \Longrightarrow & \mathcal{R}_{\mathrm{deb}}=\frac{W_{\mathrm{deb}}^{\mathrm{f}} \cdot V^{\mathrm{f}}}{A^{\mathrm{f}}}=\frac{4 \cdot V^{\mathrm{f}} \cdot \mathcal{G}_{\mathrm{SL}} \cdot l_{\mathrm{deb}}}{\phi^{\mathrm{f}}} \\
W_{\mathrm{po}}^{\mathrm{f}}=\frac{1}{2} \cdot C^{\mathrm{f}} \cdot \tau_{\mu} \cdot l_{\mathrm{po}}{ }^{2} & \Longrightarrow & \mathcal{R}_{\mathrm{po}}=\frac{W_{\mathrm{po}}^{\mathrm{f}} \cdot V^{\mathrm{f}}}{A^{\mathrm{f}}}=\frac{2 \cdot V^{\mathrm{f}} \cdot \tau_{\mu} \cdot l_{\mathrm{po}}{ }^{2}}{\phi^{\mathrm{f}}}
\end{array}\right.
$$

In Equation 1, $\phi^{\mathrm{f}}, C^{\mathrm{f}}$ and $A^{\mathrm{f}}$ are respectively the fibre diameter, perimeter and area; $V^{\mathrm{f}}$ is the fibre volume fraction, and $l_{\mathrm{deb}}$ and $l_{\mathrm{po}}$ are the debonding and pull-out lengths. The debonding component depends on the mode-II in-situ interfacial toughness $\mathcal{G}_{\mathrm{SL}}$, while the pull-out counterpart assumes constant in-situ frictional stresses $\tau_{\mu}$ (Kelly, 1970). Other formulations have included Poisson's effects (Gao et al., 1988).

Statistical models (considering fibre strength variability) have been widely used to predict the tensile strength of UD composites (Curtin, 1991, Daniels, 1945, Harlow and Phoenix, 1978a,b, Pimenta and Pinho, 2013), and some authors have extended this approach to the fracture toughness. Curtin (1993) calculated fibre pull-out lengths in ceramic-matrix composites with multiple transverse matrix cracks. Beyerlein and Phoenix (1997a,b) concluded that UD composites can be toughened by fibre strength variability, even if no pull-out is allowed. Wells and Beaumont (1982, 1985b) and Chiang (2000) have estimated debonding and pull-out toughness contributions by (i) determining the stress field along debonded fibres, and (ii) calculating the probability of failure at a distance from the main fracture plane.

However, no statistical model has considered size effects nor the intricate fracture surfaces shown in Figure 2. Wells and Beaumont (1985a) proposed the only model in the literature to acknowledge the presence of more than one scales of debonding and pull-out on the fracture surface of FRPs. However, their approach is restricted to single-fibres and bundles with known cross-section, with no interaction between the two levels.

The self-similar features shown in Figure 2 suggest that a fractal approach may be of interest. Carpinteri (1994) introduced the analogy between the fracture surface of heterogeneous materials and invasive fractals; larger structures would therefore develop more fractal levels and, consequently, present a higher apparent toughness. Different formulations based on fractal concepts have been proposed (e.g. Balankin, 1997, Borodich, 1997, Carpinteri and Chiaia, 1996, Carpinteri and Puzzi, 2009, Weiss, 
2001); specific applications include concrete (Carpinteri and Chiaia, 1995) and particlereinforced composites (Carpinteri and Paggi, 2009), but not FRPs.

Bažant (1997) and Bažant and Yavari (2005) have criticised some of the mathematical abstractions in the fractal formalism. Bažant and Kazemi (1990) proposed a scaling law based on energetic size-effects, suggesting that the apparent fracture energy of quasi-brittle materials increases with the specimen size due to the finite dimensions of their damage process zone. Their scaling law does not distinguish between dissipation mechanisms at the micro-scale, nor predicts the different fracture surfaces shown in Figure 1.

In summary, there is a striking lack of analytical models for predicting the translaminar fracture toughness of FRPs and associated size effects. Such models would be useful not only for the simulation of damage tolerant structures (Camanho et al., 2007, Chen et al., 2013, van der Meer et al., 2010, Pinho et al., 2006b), but also for understanding the toughening mechanisms and guiding material development.

This paper therefore presents an original model for the translaminar fracture toughness of FRPs, combining a stochastic model for the strength of composite bundles (Pimenta and Pinho, 2013), fracture mechanics and quasi-fractal fracture surfaces. The

model accounts for the debonding and pull-out of fibres and bundles, as well as the effect of fibre bridging and variable fibre strength.

\section{Model development}

\subsection{Geometry of quasi-fractal surfaces}

This model is based on the formation of hierarchical fracture surfaces with pulledout fibres and bundles, forming quasi-fractal (statistically self-affine, not necessarily self-similar) patterns (Figure 3). The quasi-fractal geometry results from the combination of two central assumptions: a hierarchical failure process (following most statistical Fibre Bundle Models (FBMs), e.g. Curtin, 1991, Daniels, 1945, Harlow and Phoenix, 1978a,b, Pimenta and Pinho, 2013) on the one hand, and the self-similar pull-out features in fracture surfaces (shown in Figure 2) on the other. While assuming quasi-fractal fracture surfaces (i.e. with hierarchical debonding and pull-out features, self-affine throughout a finite number of scales) is key to the model development, no other fractal formalisms are required.

Figure 3a illustrates the concept of considering hexagonal fibre packing (subscript $\mathrm{H})$; the fracture surface is hierarchically built in the following manner: 

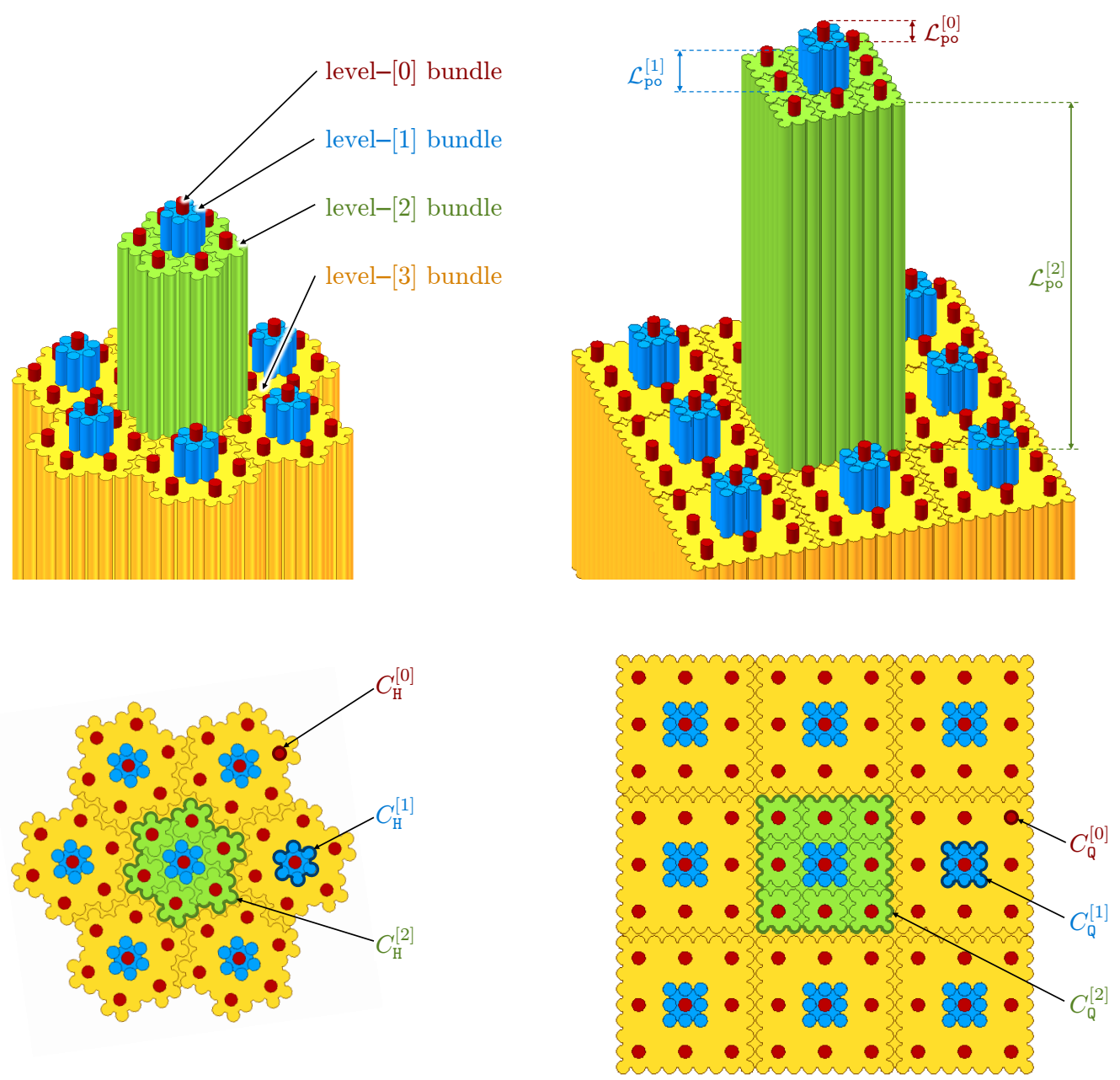

(a) Coordination number $c_{\mathcal{G}}=7$ in hexagonal fibre arrangement.

(b) Coordination number $c_{\mathcal{G}}=9$ in quadrangular fibre arrangement.

Figure 3: Quasi-fractal fracture surfaces.

- A level-[1] surface (or bundle) consists of 7 individual (level-[0]) fibres, one of which (in red) protrudes from its 6 co-planar neighbours. The number of individual fibres in the level-[1] bundle is:

$$
n_{\mathrm{H}}^{[1]}=7 ;
$$

- A level-[2] surface consists of 7 level-[1] bundles, one of which (in blue) protrudes from its 6 co-planar neighbours. The number of individual fibres in the level-[2] surface is:

$$
n_{\mathrm{H}}^{[2]}=7 \cdot n_{\mathrm{H}}^{[1]}=7^{2}=49 ;
$$


- A level-[3] surface consists of 7 level-[2] bundles, one of which (in green) protrudes from its 6 co-planar neighbours. The number of individual fibres in the level-[3] surface is:

$$
n_{\mathrm{H}}^{[3]}=7 \cdot n_{\mathrm{H}}^{[2]}=7^{3}=343
$$

- More generically, a level-[i] surface consists of 7 level- $[i-1]$ bundles, one of which protrudes from its 6 co-planar neighbours. The number of individual fibres in the level- $[i]$ surface is:

$$
n_{\mathrm{H}}^{[i]}=7 \cdot n_{\mathrm{H}}^{[i-1]}=7^{i}
$$

A fracture surface is thus characterised by the coordination number $c_{\mathcal{G}}$, which represents the number of level- $[i-1]$ bundles forming a level- $[i]$ bundle. For the hexagonal fibre packing shown in Figure $3 \mathrm{a}$ and described above, $c_{\mathcal{G}}=7$. For the square packing (subscript $\mathrm{Q}$ ) in Figure $3 \mathrm{~b}, c_{\mathcal{G}}=9$, and the number of individual fibres in each level is:

$n_{\mathrm{Q}}^{[1]}=9 ; \quad n_{\mathrm{Q}}^{[2]}=9 \cdot n_{\mathrm{Q}}^{[1]}=9^{2}=81 ; \quad n_{\mathrm{Q}}^{[3]}=9 \cdot n_{\mathrm{Q}}^{[2]}=9^{3}=729 ; \quad n_{\mathrm{Q}}^{[i]}=9 \cdot n_{\mathrm{H}}^{[i-1]}=9^{i}$.

For a generic fibre packing with coordination number $c_{\mathcal{G}}$, a level- $[i]$ surface consists of $c_{\mathcal{G}}$ level- $[i-1]$ bundles, one of which protrudes from its $c_{\mathcal{G}}-1$ co-planar neighbours. The number of individual fibres in each level- $[i]$ bundle $\left(n^{[i]}\right)$ is related to the coordination number $c_{\mathcal{G}}$ by:

$$
n^{[i]}=\left(c_{\mathcal{G}}\right)^{i} \quad \Longleftrightarrow \quad i=\log _{c_{\mathcal{G}}} n^{[i]}
$$

The total cross-section of fibres within a level- $[i]$ bundle is:

$$
A^{[i]}=n^{[i]} \cdot A^{\mathrm{f}} .
$$

Assuming preferential interfacial failure (see Figure 3), the perimeter of a level-[0] bundle $\left(C^{[0]}\right)$ is the circumference of an individual fibre, $C^{[0]}=C^{\mathrm{f}}$. Similarly, $C^{[1]}$ represents the perimeter of a protruded level-[1] bundle, and $C^{[2]}$ that of a protruded level-[2] bundle (see Figure 3). The perimeter of a generic level- $[i]$ bundle with coordination number $c_{\mathcal{G}}=7$ or $c_{\mathcal{G}}=9$ (defined by Pimenta and Pinho, 2013) is: 


$$
\left\{\begin{array}{l}
C_{\mathrm{H}}^{[i]}=3 \cdot\left(\sqrt[\log _{3} \sqrt[7]{n^{[i]}}]{n^{2}}-1\right) \cdot s_{\mathrm{H}}+\left(3 \cdot \sqrt[\log _{3} \sqrt[7]{n^{[i]}}]{ }-1\right) \cdot \frac{C^{\mathrm{f}}}{2}, \quad s_{\mathrm{H}}=\left(\sqrt{\frac{\pi}{2 \sqrt{3} V^{\mathrm{f}}}}-1\right) \cdot \phi^{\mathrm{f}} \\
C_{\mathrm{Q}}^{[i]}=3 \cdot C^{\mathrm{f}}+4 \cdot\left[\left(\sqrt{n^{[i]}}-1\right) \cdot s_{\mathrm{Q}}+\left(\sqrt{n^{[i]}}-2\right) \cdot \frac{C^{\mathrm{f}}}{2}\right], s_{\mathrm{Q}}=\left(\frac{\sqrt{\pi}}{2 \sqrt{V^{\mathrm{f}}}}-1\right) \cdot \phi^{\mathrm{f}}
\end{array}\right.
$$

This model assumes that the coordination number $c_{\mathcal{G}}$ is deterministic; consequently, all level- $[i]$ bundles have a deterministic perimeter (given by Equation 5) as well. Parametric studies (see Figure 14 in Section 3.3) will demonstrate that the predicted fracture toughness is relatively insensitive to variations within $c_{\mathcal{G}} \in\{6,7,8,9\}$, which corroborates this abstraction.

The pull-out length of a level-[0] bundle $\left(\mathcal{L}_{\text {po }}^{[0]}\right)$ is defined as the distance between the fracture surface of a protruded single fibre (in red, Figure 3 ) and that of its $c_{\mathcal{G}}-1$ level[0] neighbours. Similarly, $\mathcal{L}_{\text {po }}^{[1]}$ is the pull-out length of a level-[1] bundle, defined as distance between the main fracture surface of a protruded level-[1] bundle (in blue) and that of its surrounding neighbours. The distance between the main fracture surfaces of a protruded level-[2] bundle (in green) and that of the level-[3] bundle (in yellow) is $\mathcal{L}_{\mathrm{po}}^{[2]}$.

The pull-out length of each level-[i] bundle, $\mathcal{L}_{\mathrm{po}}^{[i]}$, is a stochastic variable; its Cumulative Distribution Function (CDF) and complementary CDF (cCDF) are respectively:

$$
\mathcal{F}_{\mathrm{po}}^{[i]}\left(l_{\mathrm{po}}^{[i]}\right)=\operatorname{Pr}\left(\mathcal{L}_{\mathrm{po}}^{[i]} \leq l_{\mathrm{po}}^{[i]}\right) \quad \text { and } \quad \mathcal{S}_{\mathrm{po}}^{[i]}\left(l_{\mathrm{po}}^{[i]}\right)=\operatorname{Pr}\left(\mathcal{L}_{\mathrm{po}}^{[i]}>l_{\mathrm{po}}^{[i]}\right)
$$

For pull-out to occur, each level-[i] pulled-out bundle debonds (splits longitudinally) from the surrounding composite; the corresponding debonding length, $\mathcal{L}_{\text {deb }}^{[i]}$ is also a stochastic variable, with:

$$
\mathcal{F}_{\mathrm{deb}}^{[i]}\left(l_{\mathrm{deb}}^{[i]}\right)=\operatorname{Pr}\left(\mathcal{L}_{\mathrm{deb}}^{[i]} \leq l_{\mathrm{deb}}^{[i]}\right) \quad \text { and } \quad \mathcal{S}_{\mathrm{deb}}^{[i]}\left(l_{\mathrm{deb}}^{[i]}\right)=\operatorname{Pr}\left(\mathcal{L}_{\mathrm{deb}}^{[i]}>l_{\mathrm{deb}}^{[i]}\right)
$$

Debonding and pull-out length distributions will be defined in the next sections.

\subsection{Formation of quasi-fractal fracture surfaces}

\subsubsection{Hierarchical failure process}

The hierarchical failure process assumed in this model relies on the following assumptions: 
A(i) Fracture propagates down (i.e. inwards) the hierarchy of a quasi-fractal surface. This follows experimental evidence of free surfaces or discontinuities triggering failure; for instance, in cross-ply CT specimens, failure starts at the $0^{\circ} / 90^{\circ}$ interface and propagates inwards to the $0^{\circ}$ plies (Laffan et al., 2010, Pinho et al., 2006a).

A(ii) Fracture occurs discretely at each hierarchical level: all level- $[i]$ breaks occur simultaneously, after failure of level $[i+1]$ and before failure of level $[i-1]$.

A(iii) Immediately after level- $[i+1]$ failure, the two fracture faces remain bridged by all the lower hierarchical levels ([0] to $[i]$ ), which share the remote load equally. While less accurate than local load sharing schemes (e.g. Beyerlein and Phoenix, 1996), this approximation is necessary to verify the discrete hierarchical failure process. Consequently, all bridging fibres undergo the same stress concentration factor $k_{\mathcal{G}}^{[i]}$ at the main level- $[i+1]$ fracture plane; $k_{\mathcal{G}}^{[i]}$ is calculated as the total-to-bridging fibre ratio.

A(iv) A level- $[i]$ pulled-out bundle is locally stronger than their neighbours; it fails therefore later and away from the surrounding fracture plane (at a distance $\mathcal{L}_{\mathrm{po}}^{[i]}$ ), after debonding bi-laterally along a length $\mathcal{L}_{\text {deb }}^{[i]}$.

The resulting sequence of events leading to complete failure of a level-[3] bundle with hexagonal fibre packing $\left(c_{\mathcal{G}}=7\right)$ is illustrated in Figure 4 and described below:

(a) Before loading (Figure $4 \mathrm{a}$ ): all $n_{\mathrm{H}}^{[3]}=c_{\mathcal{G}}{ }^{3}=343$ fibres in the bundle survive.

(b) Level-[3] failure (Figure 4b): at a certain remote load, all level-[3] fibres fail simultaneously in a planar fracture surface. The fracture surface is now partially broken while the following sub-bundles survive:

- 1 central level-[2] bundle;

- 6 level-[1] bundles surrounding the surviving level-[2] bundle;

- 6 level-[0] fibres surrounding each of the 6 surviving level-[1] bundles.

(c) Level-[2] failure (Figure 4c): the stress concentration factor (i.e. total-to-bridging fibre ratio, see $\mathbf{A}($ iii) above) after level-[3] failure is:

$$
k_{\mathcal{G}}^{[2]}=\frac{7^{3}}{1 \cdot n_{\mathrm{H}}^{[2]}+6 \cdot n_{\mathrm{H}}^{[1]}+6 \cdot 6 \cdot n_{\mathrm{H}}^{[0]}}=2.70 \quad, \quad \text { for } \quad c_{\mathcal{G}}=7 .
$$




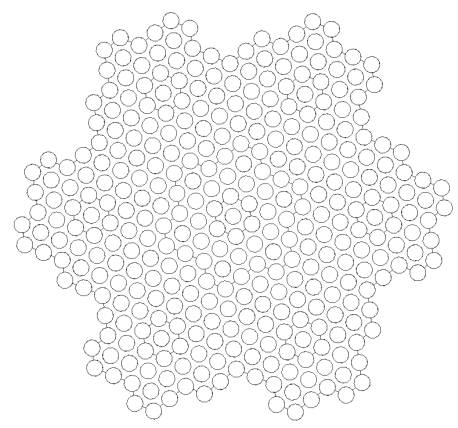

(a) Before failure onset.

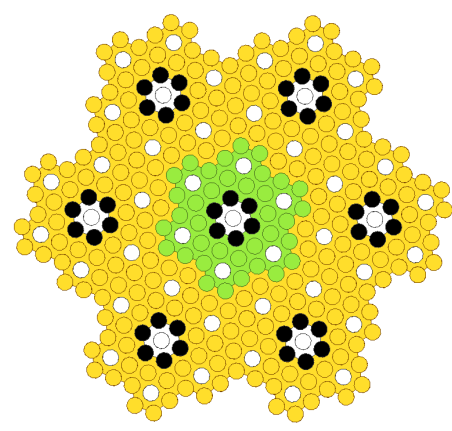

(d) Level-[1] failure.

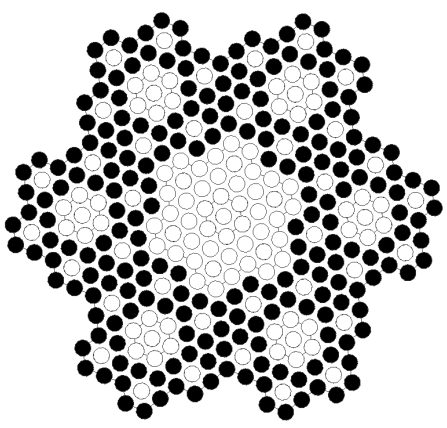

(b) Level-[3] failure.

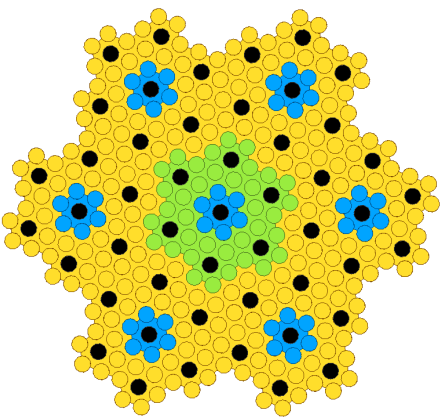

(e) Level-[0] failure.

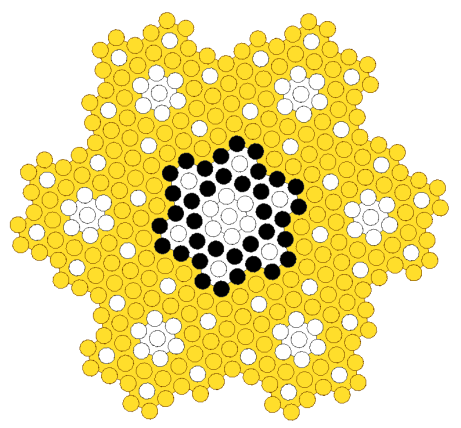

(c) Level-[2] failure.

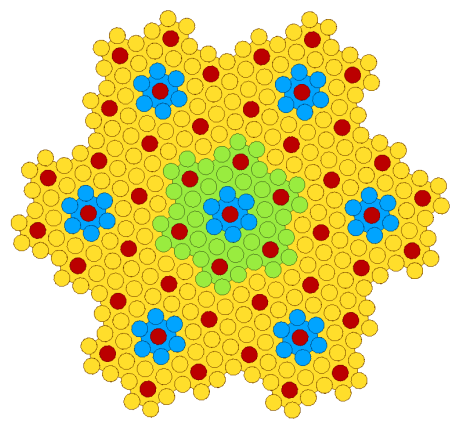

(f) Complete failure.

Figure 4: Hierarchical propagation of failure (top view, $c_{\mathcal{G}}=7$ ). Key: white circles — bridging (surviving) fibres; black circles — fibres undergoing failure; coloured circles — broken fibres.

Under the stress concentration $k_{\mathcal{G}}^{[2]}$, all level-[2] fibres within the level-[2] protruded bundle break simultaneously. The fracture surface is now composed by 7 level-[2] bundles (1 protruded, in green in Figure 3a), each partially broken but bridged by the following sub-bundles:

- 1 central level-[1] bundle;

- 6 level-[0] fibres surrounding the surviving level-[1] bundle.

(d) Level-[1] failure (Figure 4d): the stress concentration factor after level-[2] failure is:

$$
k_{\mathcal{G}}^{[1]}=\frac{7^{2}}{1 \cdot n_{\mathrm{H}}^{[1]}+6 \cdot n_{\mathrm{H}}^{[0]}}=3.77 \quad, \quad \text { for } c_{\mathcal{G}}=7 .
$$

Under the stress concentration $k_{\mathcal{G}}^{[1]}$, all level-[1] fibres within each level-[2] bundle break simultaneously. The fracture surface is now composed by $7^{2}$ level-[1] bundles ( 7 protruded, in blue in Figure 3a), each partially broken but bridged by the following sub-bundle:

- 1 central level-[0] bundle (individual fibre). 
(e) Level-[0] failure (Figure 4e): the stress concentration after level-[1] failure is:

$$
k_{\mathcal{G}}^{[0]}=\frac{7^{1}}{1 \cdot n_{\mathrm{H}}^{[0]}}=7 \quad, \quad \text { for } c_{\mathcal{G}}=7 .
$$

Each level-[0] breaks under the stress concentration $k_{\mathcal{G}}^{[0]}$, leaving the level-[3] bundle completely fractured (Figure 4f).

It is shown in Appendix A that, for a generic coordination number $c_{\mathcal{G}}$, the stress concentration factor seen by the bridging fibres when level- $[i]$ failure is about to occur is:

$$
k_{\mathcal{G}}^{[i]}=\frac{1}{1-\left(1-\frac{1}{c_{\mathcal{G}}}\right)^{i+1}} \quad, \quad \text { hence } \quad\left\{\begin{array}{l}
k_{\mathcal{G}}^{[0]}=c_{\mathcal{G}} \\
\lim _{i \rightarrow \infty} k_{\mathcal{G}}^{[i]}=1
\end{array}\right.
$$

\subsubsection{Stress fields during failure}

The evolution of stress fields during the hierarchical failure process defined in Section 2.2.1 is resolved through an axi-symmetric fracture mechanics model (Figure 5a).

Consider at first the particular case of a bundle with $c_{\mathcal{G}}=7$, just before level[1] failure (Figure 4d). Each bridging level-[1] bundle (in blue in Figure 5a, hereby represented by the superscript $\mathcal{A}$ ) is surrounded by 6 partially broken neighbours (in green in Figure 5a, hereby represented by the superscript $\mathcal{B}$ ). Each of the latter is composed by level-[2] broken fibres (forming a planar fracture surface, in green in Figure 5a) and bridging level-[0] individual fibres (in red in Figure 5a).

According to Assumption A(iii) in Section 2.2.1, all bridging fibres will be under the same stress concentration $k_{\mathcal{G}}^{[1]}$ (Equation 8 ). Consequently, the equilibrium average stresses transmitted by bundles $\mathcal{A}$ and $\mathcal{B}$ at $x=0$ are:

$$
\sigma_{0}^{\mathcal{A}}=k_{\mathcal{G}}^{[1]} \cdot \sigma^{\infty} \quad \text { and } \quad \sigma_{0}^{\mathcal{B}}=\frac{c_{\mathcal{G}}-k_{\mathcal{G}}^{[1]}}{c_{\mathcal{G}}-1} \cdot \sigma^{\infty}
$$

where $\sigma^{\infty}$ is the remote longitudinal tensile stress.

Such stress mismatch triggers a longitudinal debond at the interface between $\mathcal{A}$ (the level-[1] bridging bundle) and $\mathcal{B}$ (its partially broken neighbours), growing symmetrically from the fracture plane up to a distance $|x|=a^{[1]}$ (Figure 5b). Within the debonded region, stresses are transferred through a shear-lag mechanism with constant frictional stresses $\tau_{\mu}$; this neglects Poisson's effects, for the sake of simplicity (see justification in Appendix E). The remote stress is recovered with a discontinuity at 


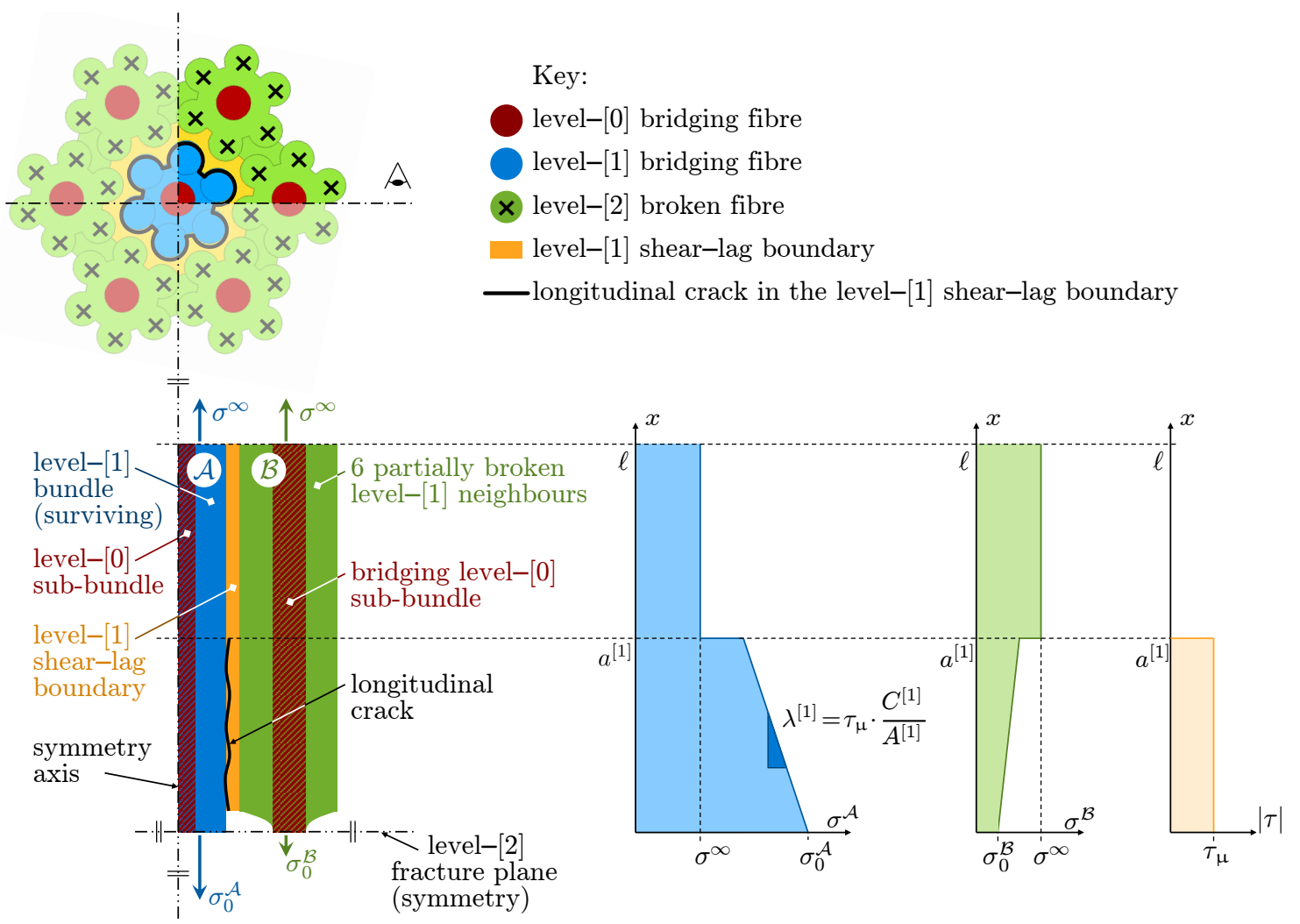

(a) Geometry.

(b) Stress fields.

Figure 5: Axi-symmetric model for level-[i] failure.

$x=a^{[1]}$, thus assuming a very small damage process zone (compared to the debonded distance). The resulting stress fields in the level-[1] bridging bundle $(\mathcal{A})$, the partiallybroken level-[2] neighbour $(\mathcal{B})$, and the $\mathcal{A}-\mathcal{B}$ interface are represented schematically in Figure 5b.

This analysis can be generalised for the configuration preceding level- $[i]$ failure (Figure 5 corresponds to $i=1$ ). In this case, the central bridging level- $[i]$ bundle $(\mathcal{A})$ is surrounded by $c_{\mathcal{G}}-1$ partially broken level- $[i]$ neighbours $(\mathcal{B})$; all bridging fibres are under the same stress concentration factor $k_{\mathcal{G}}^{[i]}$ (Equation 10). The longitudinal stress fields in bundles $\mathcal{A}$ and $\mathcal{B}$ are therefore:

$$
\sigma^{\mathcal{A}}(x)=\left\{\begin{array}{ll}
k_{\mathcal{G}}^{[i]} \cdot \sigma^{\infty}-\lambda^{[i]} \cdot x & , x \leq a^{[i]} \\
\sigma^{\infty} & , x>a^{[i]}
\end{array}, \quad \sigma^{\mathcal{B}}(x)= \begin{cases}\frac{\left(c_{\mathcal{G}}-k_{\mathcal{G}}^{[i]}\right) \cdot \sigma^{\infty}-\lambda^{[i]} \cdot x}{c_{\mathcal{G}}-1} & , x \leq a^{[i]} \\
\sigma^{\infty} & , x>a^{[i]}\end{cases}\right.
$$


where

$$
\lambda^{[i]}=\tau_{\mu} \cdot \frac{C^{[i]}}{A^{[i]}} .
$$

Imposing balance between the system's energy release rate and the in-situ mode-II debonding toughness $\mathcal{G}_{\mathrm{SL}}$, it is demonstrated in Appendix B that, at a given applied stress $\sigma^{\infty}$, the level- $[i]$ equilibrium debonding distance $a^{[i]}$ is:

$$
\begin{aligned}
a^{[i]}\left(\sigma^{\infty}\right) & =\left\{\begin{array}{ll}
0 & , \sigma^{\infty} \leq \sqrt{\psi^{[i]} / \kappa^{[i]}} \\
\frac{\left(c_{\mathcal{G}}-1\right) \cdot \sigma^{\infty}-\sqrt{\left[\left(c_{\mathcal{G}}-k_{\mathcal{G}}^{[i]}\right) \cdot \sigma^{\infty}\right]^{2}+\psi^{[i]}}}{\lambda^{[i]}}, & \sigma^{\infty}>\sqrt{\psi^{[i]} / \kappa^{[i]}}
\end{array},\right. \text { with } \\
\psi^{[i]} & =\frac{2 \cdot\left(c_{\mathcal{G}}-1\right) \cdot E^{\mathrm{f}} \cdot \mathcal{G}_{\mathrm{SL}} \cdot C^{[i]}}{c_{\mathcal{G}} \cdot A^{[i]}} \text { and } \quad \kappa^{[i]}=\left(k_{\mathcal{G}}^{[i]}-1\right) \cdot\left(2 \cdot c_{\mathcal{G}}-k_{\mathcal{G}}^{[i]}-1\right)
\end{aligned}
$$

It must be noticed that both $\mathcal{G}_{\mathrm{SL}}$ and $\tau_{\mu}$ are in-situ properties. Methods to estimate their value based on measurable properties - respectively mode-II delamination toughness $\left(\mathcal{G}_{\mathrm{II}}\right)$ and single-fibre composite interfacial friction $\left(\tau_{\mu}^{0}\right)$, obtained through Single-Fibre Pull-Out (SFPO) tests - are derived in Appendix C and Appendix D.

\subsubsection{Sequence of events for level-[i] failure}

Let $X_{\mathrm{deb}}^{[i]}$ be the stochastic strength of a level- $[i]$ bundle within the debonded length, so that failure occurs at the remote stress $\sigma^{\infty}$ within $x \in\left[-a^{[i]}\left(\sigma^{\infty}\right),+a^{[i]}\left(\sigma^{\infty}\right)\right]$ if and only if $\sigma^{\infty}=X_{\mathrm{deb}}^{[i]}$. To ensure the stress fields in Figure 5 remain valid, two possible sequences of events leading to debonding, failure and pull-out of a level- $[i]$ bundle are considered:

$E_{1}$ : Debonding followed by bundle fracture. The bundle fractures at a remote stress $\sigma^{\infty}=X_{\text {deb }}^{[i]}$, at a stochastic location $|x|=L_{\text {po }}^{[i]}$ within the debonded length $\left(L_{\mathrm{po}}^{[i]}<a^{[i]}\left(X_{\mathrm{deb}}^{[i]}\right)\right)$. This neglects the influence of breaks formed away from the level- $[i+1]$ fracture plane on the stress field in the debonded region.

$E_{2}$ : Bundle fracture followed by debonding. The bundle fractures at a remote stress $\sigma^{\infty}<X_{\text {deb }}^{[i]}$ outside the debonded length (at a location $|x|=L_{\mathrm{po}}^{[i]}>a^{[i]}\left(\sigma^{\infty}\right)$ ), but the stresses are locally recovered (and the field near the level- $[i+1]$ fracture plane is not significantly disturbed). Failure becomes effective when the debond reaches the location of fracture $\left(a^{[i]}\left(\sigma^{\infty}\right)=L_{\mathrm{po}}^{[i]}\right)$, at the remote stress $\sigma^{\infty}=X_{\mathrm{deb}}^{[i]}$.

In both cases, the realisations of debonding and pull--out lengths (see Figure 6) are: 


$$
L_{\mathrm{deb}}^{[i]}=2 \cdot a^{[i]}\left(X_{\mathrm{deb}}^{[i]}\right) \quad \text { and } \quad L_{\mathrm{po}}^{[i]} \leq a^{[i]}\left(X_{\mathrm{deb}}^{[i]}\right) \quad \Longrightarrow \quad L_{\mathrm{po}}^{[i]} \leq \frac{L_{\mathrm{deb}}^{[i]}}{2}
$$

\subsection{Determination of debonding and pull-out length distributions}

\subsubsection{Debonding length distribution}

The unequivocal relation between realisations of level- $[i]$ debonding length and level-[i] bundle strength (Equation 15) implies that the probability distributions of both variables coincide; consequently,

$$
S_{\mathrm{deb}}^{[i]}\left(l_{\mathrm{deb}}^{[i]}\right)=\operatorname{Pr}\left(L_{\mathrm{deb}}^{[i]}>l_{\mathrm{deb}}^{[i]}\right)=\operatorname{Pr}\left(X_{\mathrm{deb}}^{[i]}>\sigma^{\infty}\right) \quad \text {, with } \quad l_{\mathrm{deb}}^{[i]}=2 \cdot a^{[i]}\left(\sigma^{\infty}\right)
$$

Each distribution $S_{\mathrm{deb}}^{[i]}\left(l_{\mathrm{deb}}^{[i]}\right)$ is thus related to the bundle strength distribution for the corresponding hierarchical level.

Let $S_{\mathrm{U}, \mathrm{r}}^{[i]}(\sigma)$ be the size-dependent survival probability of a level-[i] bundle of length $l_{\mathrm{r}}$ under uniform stresses $\sigma$; this can be obtained for instance using the composite Fibre Bundle Model (FBM) previously developed by the authors (Pimenta and Pinho, 2013). To account for the non-uniform stress field within the debonded region (Figure 6a), it is convenient to define the strength distribution under a bi-lateral triangular field with peak stress $\sigma^{\max }$ and constant slope $\lambda^{[i]}$ (Figure 6c), hereby represented as $S_{\lambda}^{[i]}\left(\sigma^{\max }\right)$.

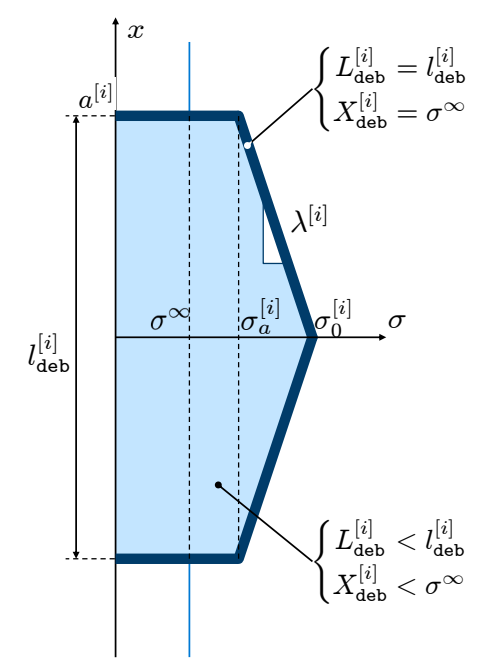

(a) Debonding length and possible location and stresses of failure.

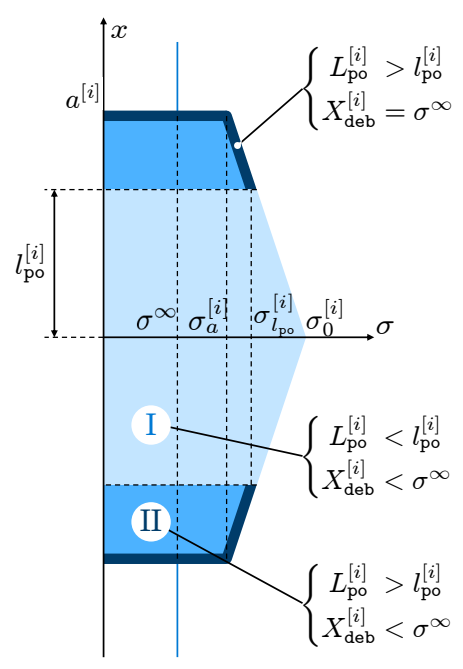

(b) Pull-out length assuming a given strength.

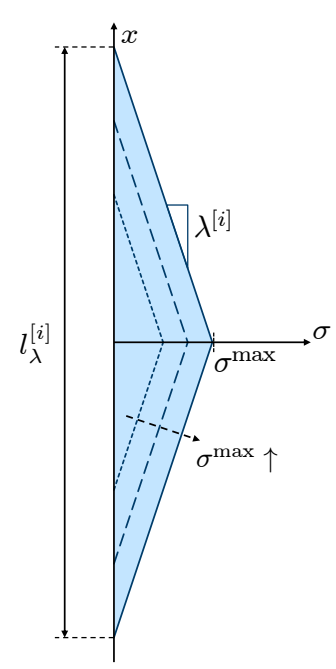

(c) Triangular stress field with frictional shear-lag.

Figure 6: Locations of failure for length distributions. 
It is shown in Appendix E that $S_{\lambda}^{[i]}\left(\sigma^{\max }\right)$ and $S_{\mathrm{U}, \mathrm{r}}^{[i]}(\sigma)$ are related by:

$$
\ln \left[S_{\lambda}^{[i]}\left(\sigma^{\max }\right)\right]=\frac{2}{\lambda^{[i] \cdot l_{\mathrm{r}}}} \cdot \int_{\sigma=0}^{\sigma^{\max }} \ln \left[S_{\mathrm{U}, \mathrm{r}}^{[i]}(\sigma)\right] \mathrm{d} \sigma
$$

Combining this with the stress field in Figure 6a, the debonding length distribution is:

$$
S_{\mathrm{deb}}^{[i]}\left(l_{\mathrm{deb}}^{[i]}\right)=\frac{S_{\lambda}^{[i]}\left(\sigma_{0}^{[i]}\left(\sigma^{\infty}\right)\right)}{S_{\lambda}^{[i]}\left(\sigma_{a}^{[i]}\left(\sigma^{\infty}\right)\right)}=\exp \left(\ln \left[S_{\lambda}^{[i]}\left(\sigma_{0}^{[i]}\left(\sigma^{\infty}\right)\right)\right]-\ln \left[S_{\lambda}^{[i]}\left(\sigma_{a}^{[i]}\left(\sigma^{\infty}\right)\right)\right]\right)
$$

where the relevant stress points are (from Equation 12):

$$
\left\{\begin{array}{l}
\sigma_{0}^{[i]}\left(\sigma^{\infty}\right)=k_{\mathcal{G}}^{[i]} \cdot \sigma^{\infty} \\
\sigma_{a}^{[i]}\left(\sigma^{\infty}\right)=k_{\mathcal{G}}^{[i]} \cdot \sigma^{\infty}-\lambda^{[i]} \cdot a^{[i]}\left(\sigma^{\infty}\right)=\left(1-c_{\mathcal{G}}+k_{\mathcal{G}}^{[i]}\right) \cdot \sigma^{\infty}+\sqrt{\left[\left(c_{\mathcal{G}}-k_{\mathcal{G}}^{[i]}\right) \cdot \sigma^{\infty}\right]^{2}+\psi^{[i]}}
\end{array}\right.
$$

\subsubsection{Pull-out length distribution}

To define the level-- $i$ ] pull--out length distribution, consider Figure $6 \mathrm{~b}$, where:

$$
\sigma_{l_{\mathrm{po}}}^{[i]}\left(\sigma^{\infty}, l_{\mathrm{po}}^{[i]}\right) \stackrel{\text { Eq. }}{=} k_{\mathcal{G}}^{[i]} \cdot \sigma^{\infty}-\lambda^{[i]} \cdot l_{\mathrm{po}}^{[i]} .
$$

Let $S_{\mathrm{I}}^{[i]}\left(\sigma^{\infty}, l_{\mathrm{po}}^{[i]}\right)$ be the bundle survival probability in Region I, and $F_{\mathrm{II}}^{[i]}\left(\sigma^{\infty}, l_{\mathrm{po}}^{[i]}\right)$ be the bundle failure probability in Region II. According to Figure 6b, the pull-out length distribution for a given strength $X_{\mathrm{deb}}^{[i]}=\sigma^{\infty}$ is:

$$
\operatorname{Pr}\left(X_{\mathrm{deb}}^{[i]}=\sigma^{\infty} \wedge L_{\mathrm{po}}^{[i]} \geq l_{\mathrm{po}}^{[i]}\right)=S_{\mathrm{I}}^{[i]}\left(\sigma^{\infty}, l_{\mathrm{po}}^{[i]}\right) \cdot \mathrm{d}\left[F_{\mathrm{II}}^{[i]}\left(\sigma^{\infty}, l_{\mathrm{po}}^{[i]}\right)\right], \quad \forall a^{[i]}\left(\sigma^{\infty}\right) \geq l_{\mathrm{po}}^{[i]}
$$

Integrating Equation 21 for all valid debonded distances $a^{[i]}$, the pull-out length distribution comes as:

$$
S_{\mathrm{po}}^{[i]}\left(l_{\mathrm{po}}^{[i]}\right)=\operatorname{Pr}\left(L_{\mathrm{po}}^{[i]}>l_{\mathrm{po}}^{[i]}\right)=\int_{a^{[i]}\left(\sigma^{\infty}\right)=l_{\mathrm{po}}^{[i]}}^{\infty} \operatorname{Pr}\left(X_{\mathrm{deb}}^{[i]}=\sigma^{\infty} \wedge L_{\mathrm{po}}^{[i]} \geq l_{\mathrm{po}}^{[i]}\right) .
$$


After some mathematical manipulation (see Appendix F), this results in:

$$
\begin{aligned}
& S_{\mathrm{po}}^{[i]}\left(l_{\mathrm{po}}^{[i]}\right)=\frac{2}{\lambda^{[i]} \cdot l_{\mathrm{r}}} \cdot \int_{\sigma^{\infty}=\sigma^{\min }}^{\infty} S_{\mathrm{deb}}^{[i]}\left(\sigma^{\infty}\right) \cdot\left(\frac{\mathrm{d} \sigma_{a}^{[i]}}{\mathrm{d} \sigma^{\infty}} \cdot \ln \left[S_{\mathrm{U}, \mathrm{r}}\left(\sigma_{a}^{[i]}\right)\right]-k_{\mathcal{G}}^{[i]} \cdot \ln \left[S_{\mathrm{U}, \mathrm{r}}\left(\sigma_{l_{\mathrm{po}}}^{[i]}\right)\right]\right) \mathrm{d} \sigma^{\infty}, \\
& \operatorname{where}\left\{\begin{array}{l}
\sigma_{a}^{[i]}=\sigma_{a}^{[i]}\left(\sigma^{\infty}\right) \\
\sigma_{l_{\mathrm{po}}^{[i]}}=\sigma_{l_{\mathrm{po}}}^{[i]}\left(\sigma^{\infty}, l_{\mathrm{po}}^{[i]}\right) \quad \text { (as defined in Equation 19), } \\
\frac{\mathrm{d} \sigma_{a}^{[i]}}{\mathrm{d} \sigma^{\infty}}=\frac{\mathrm{d} \sigma_{a}^{[i]}}{\mathrm{d} \sigma^{\infty}}\left(\sigma^{\infty}\right)=1-c_{\mathcal{G}}+k_{\mathcal{G}}^{[i]}+\frac{\left(c_{\mathcal{G}}-k_{\mathcal{G}}^{[i]}\right)^{2} \cdot \sigma^{\infty}}{\sqrt{\left[\left(c_{\mathcal{G}}-k_{\mathcal{G}}^{[i]}\right) \cdot \sigma^{\infty}\right]^{2}+\psi^{[i]}}}, \\
\sigma^{\min }=\sigma^{\min }\left(l_{\mathrm{po}}^{[i]}\right)=\frac{\lambda^{[i]} \cdot l_{\mathrm{po}}^{[i]}}{\kappa^{[i]}} \cdot\left(c_{\mathcal{G}}-1+\sqrt{\frac{\psi^{[i]} \cdot \kappa^{[i]}}{\left(\lambda^{[i]} \cdot l_{\mathrm{po}}^{[i]}\right)^{2}}+\left(c_{\mathcal{G}}-k_{\mathcal{G}}^{[i]}\right)^{2}}\right) .
\end{array}\right.
\end{aligned}
$$

\subsubsection{Extreme value length distributions for quasi-fractal fracture surfaces}

The quasi-fractal configuration of fracture surfaces (Figure 3 and Section 2.1) assumes that, for each level $[i]$, only one out of $c_{\mathcal{G}}$ bundles debonds and pulls-out significantly, while the remaining $\left(c_{\mathcal{G}}-1\right)$ neighbours present much smaller features. Therefore, the best estimates of debonding and pull-out lengths for the protruded bundles in the idealised fracture surfaces are not the expected distributions (as calculated in Equations 18 and 22, represented by Roman variables), but their extreme value distributions (as previously defined in Equation 6, represented by Cursive variables). Mathematically, if $j$ corresponds to a level- $[i]$ bundle within a level- $[i+1]$ surface,

$$
\mathcal{L}_{\mathrm{deb}}^{[i]}=\max \left\{L_{\mathrm{deb}}^{[i], j}\right\} \quad \text { and } \quad \mathcal{L}_{\mathrm{po}}^{[i]}=\max \left\{L_{\mathrm{po}}^{[i], j}\right\} \quad, \quad \forall j \in\left\{1, \cdots, c_{\mathcal{G}}\right\}
$$

The extreme distributions are thus equal to the joint probability of the $c_{\mathcal{G}}$ indepen-

dent and identically distributed length distributions, $\operatorname{Prob}\left(\mathcal{L}^{[i]}<l^{[i]}\right)=\left[\operatorname{Prob}\left(L^{[i]}<l^{[i]}\right)\right]^{c_{\mathcal{G}}}$. Consequently, and following the distributions defined in Equations 6, 18 and 22, the CDFs of debonding and pull-out lengths in a quasi-fractal surface are:

$$
\mathcal{F}_{\mathrm{deb}}^{[i]}\left(l_{\mathrm{deb}}^{[i]}\right)=\left[1-S_{\mathrm{deb}}^{[i]}\left(l_{\mathrm{deb}}^{[i]}\right)\right]^{c_{\mathcal{G}}} \quad \text { and } \quad \mathcal{F}_{\mathrm{po}}^{[i]}\left(l_{\mathrm{po}}^{[i]}\right)=\left[1-S_{\mathrm{po}}^{[i]}\left(l_{\mathrm{po}}\right)\right]^{c_{\mathcal{G}}}
$$

\subsection{Fracture toughness of quasi-fractal fracture surfaces}

The fracture toughness of UD composites is calculated as the energy dissipated by forming quasi-fractal fracture surfaces as shown in Figure 3. This considers the contributions of (i) fibre-matrix debonding and matrix fracture, and (ii) friction during fibre 
and bundle pull-out (neglecting Poisson's effects); for each level-[i] bundle debonded and pulled-out, the expected values for energy dissipated are (Kim and Mai, 1991):

$$
W_{\mathrm{deb}}^{[i]}=C^{[i]} \cdot \mathcal{G}_{\mathrm{SL}} \cdot \mathcal{L}_{\mathrm{deb}, \mathrm{m}}^{[i]} \quad \text { and } \quad W_{\mathrm{po}}^{[i]}=\frac{1}{2} \cdot C^{[i]} \cdot \tau_{\mu} \cdot\left[\left(\mathcal{L}_{\mathrm{po}}^{[i]}\right)^{2}\right]_{\mathrm{m}}
$$

where $\mathcal{G}_{\mathrm{SL}}$ and $\tau_{\mu}$ are in-situ properties (see Appendix $\mathrm{C}$ and Appendix D). The subscript $m$ represents the mean value; $\left[\left(\mathcal{L}_{\mathrm{po}}^{[i]}\right)^{2}\right]_{\mathrm{m}}=\left(\mathcal{L}_{\mathrm{po}, \mathrm{m}}^{[i]}\right)^{2} \cdot\left[1+\left(\mathrm{CoV}_{\mathcal{L}_{\mathrm{po}}}^{[i]}\right)^{2}\right]$, being $\mathrm{CoV}$ the Coefficient of Variance.

The quasi-fractal arrangement of fracture surfaces implies that one level- $[i]$ bundle debonds and pulls-out from each level- $[i+1]$ bundle. The toughness components for each level are therefore:

$$
g_{\mathrm{deb}}^{[i]}=\frac{W_{\mathrm{deb}}^{[i]}}{c_{\mathcal{G}} \cdot A^{[i]} / V^{\mathrm{f}}} \quad \text { and } \quad g_{\mathrm{po}}^{[i]}=\frac{W_{\mathrm{po}}^{[i]}}{c_{\mathcal{G}} \cdot A^{[i]} / V^{\mathrm{f}}} \quad, \quad \text { with } \quad g^{[i]}=g_{\mathrm{deb}}^{[i]}+g_{\mathrm{po}}^{[i]} .
$$

The (expected) total toughness $\mathcal{G}$ is additive both regarding all hierarchical levels formed (from $i=0$ to $i=i^{\max }$ ) and the debonding and pull-out components,

$$
\mathcal{G}^{\left[i^{\max }\right]}=\sum_{i=0}^{i_{\max }} g^{[i]}=\mathcal{G}_{\mathrm{deb}}^{\left[i^{\max }\right]}+\mathcal{G}_{\mathrm{po}}^{\left[i_{\max }\right]} \quad \text { with } \quad \mathcal{G}_{\mathrm{deb}}^{\left[i^{\max }\right]}=\sum_{i=0}^{i_{\max }} g_{\mathrm{deb}}^{[i]} \quad \text { and } \quad \mathcal{G}_{\mathrm{po}}^{\left[i^{\max }\right]}=\sum_{i=0}^{i^{\max }} g_{\mathrm{po}}^{[i]}
$$

The number of hierarchical levels formed in a fracture surface $\left(i^{\max }\right)$ can be calculated depending on the filament count $n^{[i]}$ (Equation 3) and its configuration (Table 1); this results in a size effect on the fracture toughness of UD composites.

\subsection{Numerical implementation}

Figure 7 summarises the numerical implementation of the present model; the fol-

\begin{tabular}{|c|c|c|}
\hline Type of surface & Filament count & $i^{\max }$ \\
\hline Isolated bundle ${ }^{(\star)}$ & $n^{\max }$ fibres in the cross-section & $\log _{c_{\mathcal{G}}}\left(n^{\max }\right)-1$ \\
\hline Embedded bundle ${ }^{(\dagger)}$ & $n^{\max }$ fibres in the cross-section & $\log _{c_{\mathcal{G}}}\left(n^{\max }\right)$ \\
\hline Embedded UD ply ${ }^{(\dagger)}$ & $\sqrt{n^{\max }}$ fibres across the thickness & $\log _{c_{\mathcal{G}}}\left(n^{\max }\right)$ \\
\hline
\end{tabular}
lowing points should be underlined:

Table 1: Number of hierarchical levels $i^{\max }$ for difference types of fracture surface. 


\section{Definition of input variables}

\begin{tabular}{|c|c|c|c|}
\hline $\begin{array}{l}\text { I.1: Numerical } \\
\qquad\left\{\Delta \sigma, n_{\boldsymbol{\sigma}}, \epsilon_{\mathrm{po}}\right\}\end{array}$ & $\begin{array}{l}\text { I.2: Fibre properties } \\
\qquad\left\{l_{\mathrm{r}}, \sigma_{0}^{\mathrm{f}}, m, E^{\mathrm{f}}\right\}\end{array}$ & $\begin{array}{l}\text { I.3: Interface properties } \\
\qquad\left\{\tau_{\mu}, \mathcal{G}_{\mathrm{SL}}\right\} \text { or }\left\{\tau_{\mu}^{0}, \mathcal{G}_{\mathrm{II}}\right\}\end{array}$ & $\begin{array}{l}\text { I.4: Composite properties } \\
\qquad\left\{V^{\mathrm{f}}, \phi^{\mathrm{f}}, n^{\max }, c_{\mathcal{G}}\right\}\end{array}$ \\
\hline
\end{tabular}

I.5: $\begin{gathered}\text { Bundle stresses } \\ \text { and strengths }\end{gathered} \mathbf{S}_{\mathbf{U r}}^{[\mathbf{n}]}=\left\{S_{\mathrm{U}, \mathrm{r}}^{\left[n\left(i_{X}\right)\right]}(\sigma)\right\}$ where $\boldsymbol{\sigma}=\{(j-1) \cdot \Delta \sigma\}_{j=1}^{n_{\boldsymbol{\sigma}}}$ and $\mathbf{i}_{\mathbf{X}}=\left\{0, \cdots, i_{X}^{\max }\right\},\left\{\begin{array}{l}n_{X}(0)=1 \\ n_{X}\left(i_{X}^{\max }\right)=n^{\max }\end{array}\right.$

\section{Preliminary calculations}

II.1: Bundle geometry

$$
\begin{array}{ll}
A^{\mathrm{f}}=\pi \cdot \frac{\left(\phi^{\mathrm{f}}\right)^{2}}{4} & A^{\left[\mathbf{i}_{\mathbf{X}}\right]}=\mathbf{n}_{\mathbf{X}} \cdot A^{\mathrm{f}} \quad s_{\mathrm{SL}}=\left(\sqrt{\frac{\pi}{2 \cdot \sqrt{3} \cdot V^{\mathrm{f}}}}-1\right) \cdot \phi^{\mathrm{f}} \\
C^{\mathrm{f}}=\pi \cdot \phi^{\mathrm{f}} & C^{\left[\mathbf{i}_{\mathbf{X}}\right]}=3 \cdot\left(\sqrt\left[\log _{3} \sqrt[7]{\mathbf{n}_{\mathbf{X}}}-1\right) \cdot s_{\mathrm{SL}}\right]{ }+\left(3 \cdot \sqrt[\log _{3} \sqrt[7]{\mathbf{n}_{\mathbf{X}}}]{ }-1\right) \cdot \frac{C^{\mathrm{f}}}{2}
\end{array}
$$

\section{II.2: Strength distributions}

$$
\begin{aligned}
& \ln \mathbf{S}_{\mathbf{U r}}^{[\mathbf{i}]}=\ln \left(\mathbf{S}_{\mathbf{U r}}^{[\mathbf{i} \mathbf{x}]}\right) \\
& \ln \mathbf{S}_{\mathbf{L r}}^{[\mathbf{i} \mathbf{x}]}=\frac{1}{\sigma} \cdot \int^{\boldsymbol{\sigma}} \ln \mathbf{S}_{\mathbf{U r}}^{\left[\mathbf{i x}_{\mathbf{r}}\right]} \cdot \Delta \sigma
\end{aligned}
$$

$$
\begin{aligned}
& \forall i_{X} \in \mathbf{i}_{\mathbf{X}} \quad \text { III. Debonding and pull-out lengths and toughnesses at strength levels } \\
& \begin{array}{l}
\text { III.1: Strength-level constants } \\
i_{\mathcal{G}}=\log _{c_{\mathcal{G}}}\left[n\left(i_{X}\right)\right] \quad \quad \lambda=\tau_{\mu} \cdot \frac{\left.C^{[i x}\right]}{A^{\left[i_{x}\right]}} \quad \quad k_{\mathcal{G}}=\frac{1}{1-\left(1-1 / c_{\mathcal{G}}\right)^{i_{\mathcal{G}}+1}} \quad \psi=\frac{2 \cdot\left(c_{\mathcal{G}}-1\right) \cdot E^{\mathrm{f}} \cdot \mathcal{G}_{\mathrm{SL}} \cdot C^{\left[i_{X}\right]}}{c_{\mathcal{G}} \cdot A^{\left.i_{X}\right]}}
\end{array} \\
& \text { III.2: Debonding length distributions } \\
& \text { (vector indices } j \in\left[j_{\mathrm{a}}^{\min }, j_{\mathrm{a}}^{\max }\right] \text { ) } \\
& j_{\mathrm{a}}^{\max }=\left\lfloor\frac{n_{\sigma}-1}{k_{\mathcal{G}}}\right\rfloor+1 \\
& j_{\mathrm{a}}^{\min }=\left\lceil\frac{\sqrt{\psi /\left[\left(k_{\mathcal{G}}-1\right) \cdot\left(2 \cdot c_{\mathcal{G}}-k_{\mathcal{G}}-1\right)\right]}}{\Delta \sigma}\right\rceil+1 \\
& \ln \mathbf{S}_{\boldsymbol{\lambda}}=\frac{2 \cdot \boldsymbol{\sigma}}{\lambda \cdot l_{\mathbf{r}}} \cdot \ln \mathbf{S}_{\mathbf{L r}}^{\left[i_{\mathbf{r}}\right]} \quad \begin{array}{l}
\boldsymbol{\sigma}_{\mathbf{a}}=\left(1-c_{\mathcal{G}}+k_{\mathcal{G}}\right) \cdot \boldsymbol{\sigma}+\sqrt{\left[\left(c_{\mathcal{G}}-k_{\mathcal{G}}\right) \cdot \boldsymbol{\sigma}\right]^{2}+\psi} \\
\boldsymbol{\sigma}_{\mathbf{0}}=k_{\mathcal{G}} \cdot \boldsymbol{\sigma}
\end{array} \\
& \mathbf{a}=\frac{\left(c_{\mathcal{G}}-1\right) \cdot \boldsymbol{\sigma}-\sqrt{\left[\left(c_{\mathcal{G}}-k_{\mathcal{G}}\right) \cdot \boldsymbol{\sigma}\right]^{2}+\psi}}{\lambda} \\
& \mathbf{S}_{\mathbf{d e b}}^{[i x]}=\left\{1,\left\{\exp \left(\operatorname{lnS}_{\boldsymbol{\lambda}}\left[@ \boldsymbol{\sigma}_{\mathbf{0}}(j)\right]-\mathbf{l n S}_{\boldsymbol{\lambda}}\left[@ \boldsymbol{\sigma}_{\mathbf{a}}(j)\right]\right)\right\}_{j=j_{\mathbf{a}}^{\min }}^{j_{\mathbf{m}}^{\max }}\right\} \\
& \mathbf{l}_{\mathbf{d e b}}^{[i x]}=\left\{0,\{2 \cdot \mathbf{a}(j)\}_{j=j_{\mathbf{a}}^{\min }}^{j_{\mathbf{m}}^{\max }}\right\}
\end{aligned}
$$

III.3: Pull-out length distributions (vector indices $j \in\left[j_{\mathrm{a}}^{\min }, j_{\mathrm{po}}^{\max }\right]$ ) $\quad j_{\mathrm{po}}^{\max }=\min \left\{j: \mathbf{S}_{\mathrm{deb}}^{\left[i_{\mathrm{X}}\right]}(j)<\epsilon_{\mathrm{po}}\right\}+1$

$$
\begin{aligned}
& \Delta \boldsymbol{\sigma}_{\mathbf{a}}=1-c_{\mathcal{G}}+k_{\mathcal{G}}+\frac{\left(c_{\mathcal{G}}-k_{\mathcal{G}}\right)^{2} \cdot \boldsymbol{\sigma}}{\sqrt{\left[\left(c_{\mathcal{G}}-k_{\mathcal{G}}\right) \cdot \boldsymbol{\sigma}\right]^{2}+\psi}} \quad \quad \boldsymbol{\Sigma}_{\mathrm{I}}=\frac{2}{\lambda \cdot l_{\mathbf{r}}} \cdot \int_{j=j_{\mathrm{a}}^{\min }}^{j_{\mathrm{jo}}^{\max }} \Delta \boldsymbol{\sigma}_{\mathbf{a}} \cdot \mathbf{S}_{\mathbf{d e b}}^{[i \boldsymbol{X}]}(j) \cdot \ln \mathbf{S}_{\mathbf{U r}}^{[i \boldsymbol{X}]}\left[@ \boldsymbol{\sigma}_{\mathbf{a}}(j)\right] \cdot \Delta \sigma \\
& \forall j \in\left\{j_{\mathrm{a}}^{\min }, \cdots, j_{\mathrm{po}}^{\max }\right\} \\
& \boldsymbol{\sigma}_{\mathrm{po}}=\boldsymbol{\sigma}_{0}-\lambda_{\mu} \cdot \mathbf{a}(j) \\
& \boldsymbol{\Sigma}_{\mathrm{II}}(j)=\frac{2 \cdot k_{\mathcal{G}}}{\lambda \cdot l_{\mathbf{r}}} \cdot \int_{k=j}^{j_{\mathrm{po}}^{\max }} \mathbf{S}_{\mathbf{d e b}}^{\left[i_{X}\right]}(k) \cdot \ln \mathbf{S}_{\mathbf{U r}}^{\left[i_{X}\right]}\left[@ \sigma_{\mathbf{p o}}(k)\right] \cdot \Delta \sigma \\
& \mathbf{S}_{\mathbf{p O}}^{\left[i_{X}\right]}=\left\{1,\left\{\boldsymbol{\Sigma}_{\mathrm{I}}(j)-\boldsymbol{\Sigma}_{\mathrm{II}}(j)\right\}_{j=j_{\mathbf{a}}^{\min }}^{j_{\mathrm{po}}^{\max }}\right\} \\
& \mathbf{l}_{\mathbf{p o}}^{\left[i_{X}\right]}=\left\{0,\{\mathbf{a}(j)\}_{j=j_{\mathbf{a}}^{\min }}^{j_{\mathbf{m}}^{\max }}\right\}
\end{aligned}
$$

III.4: Expected lengths and toughnesses at strength levels

$$
\begin{aligned}
& \mathcal{F}_{\text {deb }}^{\left[i_{X}\right]}=\left(1-\mathbf{S}_{\text {deb }}^{\left[i_{X}\right]}\right)^{c_{\mathcal{C}}} \\
& \mathcal{L}_{\mathrm{deb}, \mathrm{m}}^{\left[i_{X}\right]}=\int \mathrm{l}_{\mathrm{deb}}^{\left[i_{X}\right]} \cdot \Delta \mathcal{F}_{\mathrm{deb}}^{\left[i_{X}\right]} \\
& \mathcal{F}_{\mathbf{p O}}^{\left[i_{\mathbf{X}}\right]}=\left(1-\mathbf{S}_{\mathbf{p o}}^{\left[i_{\mathbf{X}}\right]}\right)^{\mathcal{C}_{\mathcal{G}}} \\
& {\left[\left(\mathcal{L}_{\mathbf{p o}}^{\left[i_{\mathbf{X}}\right]}\right)^{2}\right]_{\mathrm{m}}=\int\left(\mathbf{l}_{\mathbf{p O}}^{\left[i_{\mathbf{X}}\right]}\right)^{2} \cdot \Delta \mathcal{F}_{\mathbf{p o}}^{\left[i_{X}\right]}} \\
& g_{\mathrm{deb}}^{\left[i_{X}\right]}=\frac{C^{\left[i_{X}\right]} \cdot \mathcal{G}_{\mathrm{SL}} \cdot \mathcal{L}_{\mathrm{deb}, \mathrm{m}}^{\left[i_{X}\right]}}{c_{\mathcal{G}} \cdot A^{\left[i_{X}\right]} / V^{\mathrm{f}}} \\
& g_{\mathrm{po}}^{\left[i_{X}\right]}=\frac{C^{\left[i_{X}\right]} \cdot \tau_{\mu} \cdot\left[\left(\mathcal{L}_{\mathrm{po}}^{\left[i_{X}\right]}\right)^{2}\right]_{\mathrm{m}}}{2 \cdot c_{\mathcal{G}} \cdot A^{\left[i_{X}\right]} / V^{f}}
\end{aligned}
$$

\section{Fracture toughnesses at the fracture surface's hierarchical levels}

$$
\begin{gathered}
\text { IV.1: Level } 0 \\
n_{\mathcal{G}}^{[0]}=1 \\
\mathcal{G}_{\text {deb }}^{[0]}=g_{\text {deb }}^{[0]}+g_{\mathrm{po}}^{[0]}
\end{gathered}
$$

IV.2: General level

$i_{\mathcal{G}}^{\max }=\left\lfloor\log _{c \mathcal{G}}\left(n^{\max }\right)\right\rfloor$

$$
\begin{aligned}
& \forall i_{\mathcal{G}} \in\left\{1, \cdots, i_{\mathcal{G}}^{\max }\right\} \\
& n_{\mathcal{G}}^{\left[i_{\mathcal{G}}\right]}=\left(c_{\mathcal{G}}\right)^{i_{\mathcal{G}}} \quad \mathcal{G}^{\left[i_{\mathcal{G}}\right]}=\mathcal{G}^{\left[i_{\mathcal{G}}-1\right]}+g_{\mathrm{deb}}^{\left[@ n_{\mathcal{G}}^{\left[i_{\mathcal{G}}\right]}\right]}+g_{\mathrm{po}}^{\left[@ n_{\mathcal{G}}^{\left[i_{\mathcal{G}}\right]}\right]}
\end{aligned}
$$

Figure 7: Overview of numerical implementation (level superscripts $\left(\left[i_{X}\right]\right.$ or $\left.\left[i_{\mathcal{G}}\right]\right)$ are omitted for all internal variables). 
- The toughness model relies on strength distributions for bundles of different filament counts $\left\{n_{X}^{[0]}=0, \cdots, n_{X}^{\left[i_{X}\right]}, \cdots, n_{X}^{\left[i_{X}^{\max }\right]}=n^{\max }\right\}$, identified by the strength level $i_{X}$. The corresponding toughness level $i_{\mathcal{G}} \equiv i$ (with coordination number $c_{\mathcal{G}}$ ) is determined from $n_{X}^{\left[i_{X}\right]}$ (Equation 3), and may not be an integer.

- Consequently, Module III calculates debonding and pull-out length distributionsfor each strength level $\left[i_{X}\right]$ (Equations 18, 22 and 24). Module IV interpolates the corresponding level- $\left[i_{X}\right]$ toughnesses (Equations 25 and 26) to integer toughness levels $i_{\mathcal{G}}$, and calculates the global toughness $\mathcal{G}^{\left[{ }^{i \mathcal{G}}\right]}$ (Equation 27);

- The independent variable for all stochastic distributions is the remote stress $\sigma^{\infty}$, discretised in the vector $\boldsymbol{\sigma}$ (with $n_{\boldsymbol{\sigma}}$ values and fixed increment step $\Delta \sigma$ ). Dependent variables are expressed in vectorial form, indicated in upright bold.

- Figure 7 makes use of array programming (e.g. MATLAB), which greatly simplifies the implementation and reduces running time. Consequently, all arithmetic operators represent pointwise calculations, and integration symbols correspond to numerical integration; interpolations are represented by the operator @.

- Determining the pull-out length distribution requires evaluating the integral in Equation 22 for each value of $l_{\mathrm{po}}$. In order to reduce the associated computational cost, this includes only those stress states for which the survival probability within the debonded length is above a given threshold $\epsilon_{\mathrm{po}}$.

The present implementation uses bundle survival distributions $\left(S_{\mathrm{U}, \mathrm{r}}^{[i \mathrm{X}]}(\sigma)\right)$ calculated through the composite FBM previously developed by the authors (Pimenta and Pinho, 2013). Although other formulations could be used, this model introduced the original concept of self-similar fracture surfaces (by including size effects in the damage process zone), and generates strength distributions for bundles with 1 to $10^{6}$ filaments in less than one second - which makes it ideal for this application. Because Pimenta and Pinho's (2013) strength model uses a coordination number of 2, the correspondence between filament count, strength-levels and toughness-levels is given by:

$$
n_{X}^{[i]}=2^{i_{X}} \quad \Longrightarrow \quad i_{X}=i_{\mathcal{G}} \cdot \log _{2}\left(c_{\mathcal{G}}\right)
$$


Table 2: Nominal model inputs for parametric studies (nominal outputs will be highlighted as $\diamond$ ).

\begin{tabular}{ccc|ccc|ccc|ccc}
\hline \multicolumn{3}{c|}{ Numerical } & \multicolumn{3}{c|}{ Fibre } & \multicolumn{3}{c|}{ Interface } & \multicolumn{3}{c}{ Composite } \\
\hline$n_{\sigma}$ & $\Delta \sigma$ & $\epsilon_{\mathrm{po}}$ & $l_{\mathrm{r}}$ & $X_{\mathrm{m}}^{\mathrm{f}}$ & $\mathrm{CoV}_{X}^{\mathrm{f}}$ & $\tau_{\mathrm{SL}}{ }^{(\dagger)}$ & $\mathcal{G}_{\mathrm{II}}$ & $\tau_{\mu}^{0}$ & $\phi^{\mathrm{f}}$ & $V^{\mathrm{f}}$ & $c_{\mathcal{G}}$ \\
$\left(10^{3}\right)$ & $(\mathrm{MPa})$ & $(-)$ & $(\mathrm{mm})$ & $(\mathrm{GPa})$ & $(\%)$ & $(\mathrm{MPa})$ & $\left(\mathrm{kJ} / \mathrm{m}^{2}\right)$ & $(\mathrm{MPa})$ & $(\mu \mathrm{m})$ & $(\%)$ & $(-)$ \\
50 & 1 & $10^{-8}$ & 10 & $4.5^{(\star)}$ & $25^{(\star)}$ & 70 & 1.0 & 10 & 5 & 60 & 7 \\
\hline
\end{tabular}

(*) Corresponding to $\sigma_{0}^{\mathrm{f}}=4.93 \mathrm{GPa}$ and $m=4.54$.

(†) Interfacial shear-lag strength (for strength model, Pimenta and Pinho, 2013).

\section{Results}

\subsection{Analysis of model predictions}

Figure 8 presents an overview of the results obtained with the toughness model, considering the nominal inputs given in Table 2. All hierarchical levels $[i]$ correspond to toughness levels, thus with $n^{[i]}=\left(c_{\mathcal{G}}\right)^{i_{\mathcal{G}}}$.

Bundle strength distributions, obtained with the model previously developed by Pimenta and Pinho (2013), are shown in Figure 8a. Figure 8b evidences size effects on the toughness, which increases considerably with filament count. The relative contributions of debonding and pull-out components is also affected: the former dominates in thinner bundles, but the latter progressively gains importance as size increases.

Larger bundles are toughened by the presence of more levels in their fracture surface, each making a positive contribution ( $g^{[i]}$ in Figure 8c) to the overall toughness. Nevertheless, this contribution becomes progressively smaller for very large bundles (see for instance $g_{\mathrm{deb}}^{[i]}$ decreasing for $i>4$ in Figure 8c).

Figure 8d presents the mean value of debonding and pull-out lengths for each hierarchical level. Their stochastic distributions are shown in Figures 8e and 8f, as functions of the corresponding aspect ratios (relative to the equivalent bundle thickness $t^{[i]}$ ):

$$
\alpha_{\mathrm{deb}}^{[i]}=\frac{l_{\mathrm{deb}}^{[i]}}{t^{[i]}} \quad \text { and } \quad \alpha_{\mathrm{po}}^{[i]}=\frac{l_{\mathrm{po}}^{[i]}}{t^{[i]}}, \quad \text { with } \quad t^{[i]}=\sqrt{\frac{A^{[i]}}{V^{\mathrm{f}}}} .
$$

\subsection{Convergence study}

Figure 9 shows that calculated fracture toughnesses converges for $\Delta \sigma \rightarrow 0$ and $\epsilon_{\mathrm{po}} \rightarrow 0$, validating the numerical implementation (Section 2.5). Numerical errors are higher for the lower levels; nonetheless, due to their minor contribution for the toughness of larger bundles, the effect of the errors vanishes upwards in the hierarchy. Converged sets of toughnesses and length distributions are computed in less than one minute. 


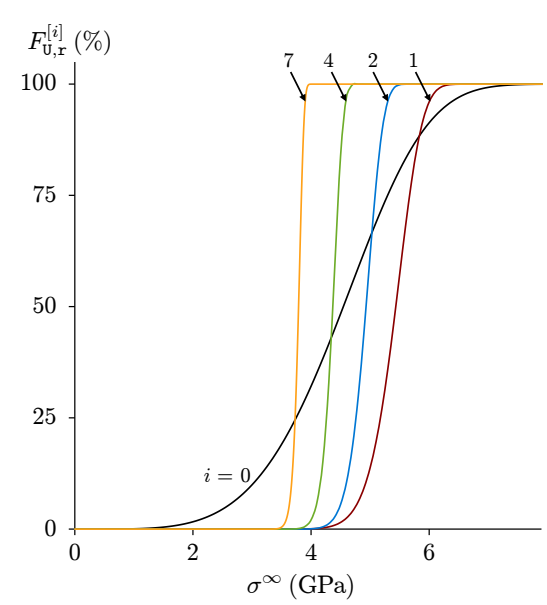

(a) Bundle strength distributions, $l_{\mathrm{r}}=10 \mathrm{~mm}$ (Pimenta and Pinho, 2013).

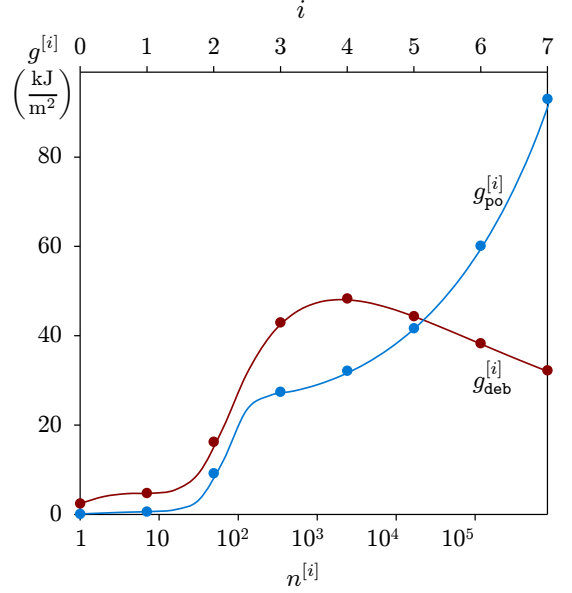

(c) Toughness contribution from each bundle level.

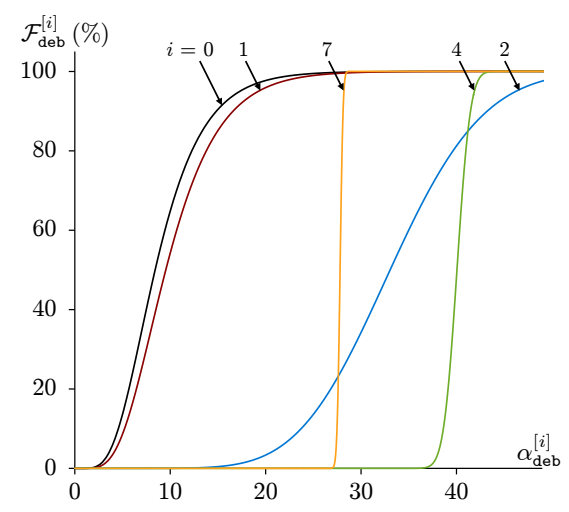

(e) Debonding aspect ratio distributions (for several approximate bundle levels $i$ ).

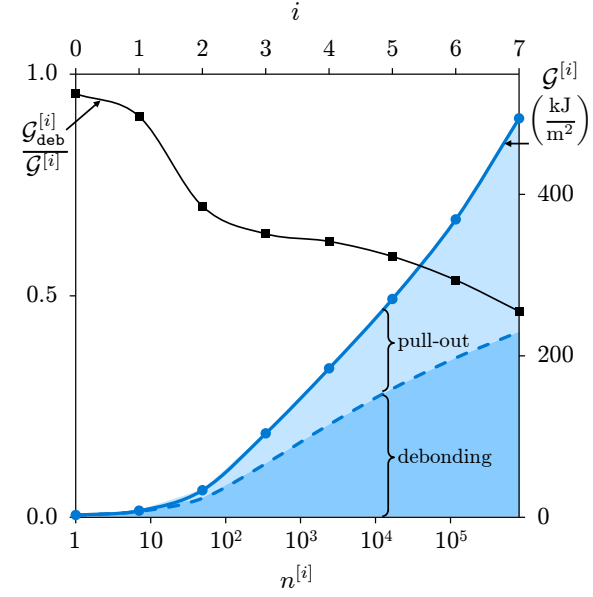

(b) Size effect on the fracture toughness.

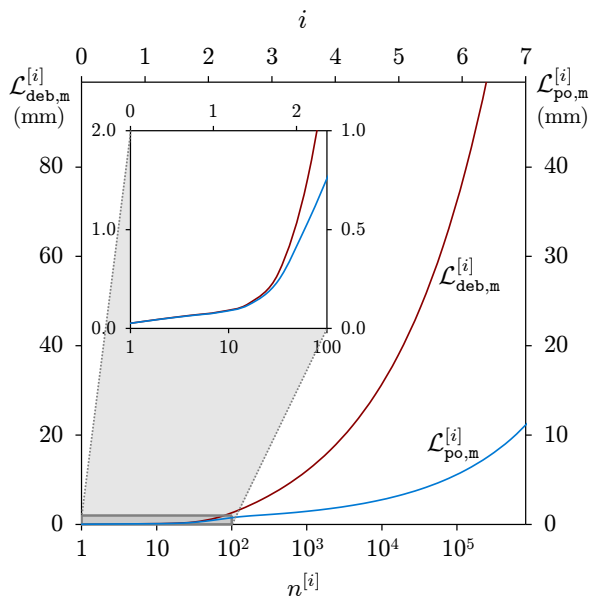

(d) Size effect on average debonding and pull-out lengths.

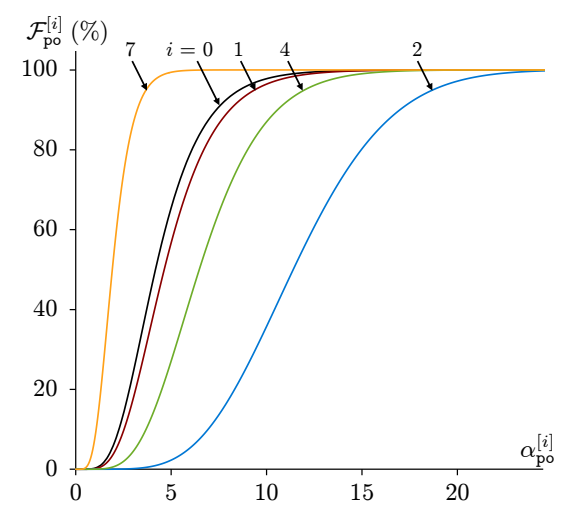

(f) Pull-out aspect ratio distributions (for several approximate bundle levels $i$ ).

Figure 8: Overview of model results. 


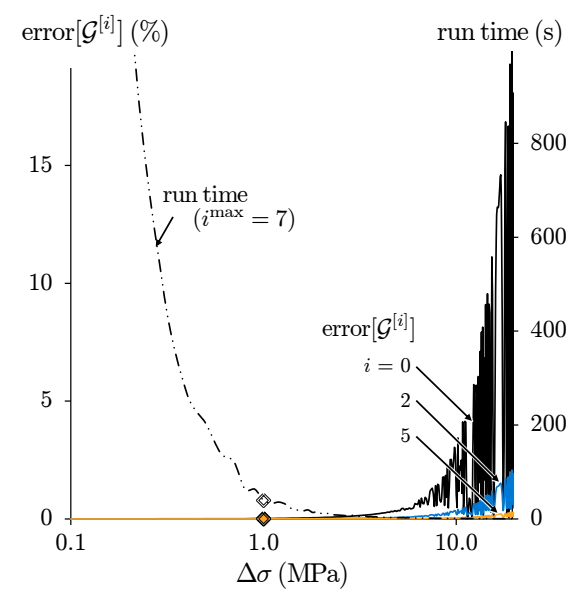

(a) Increment step.

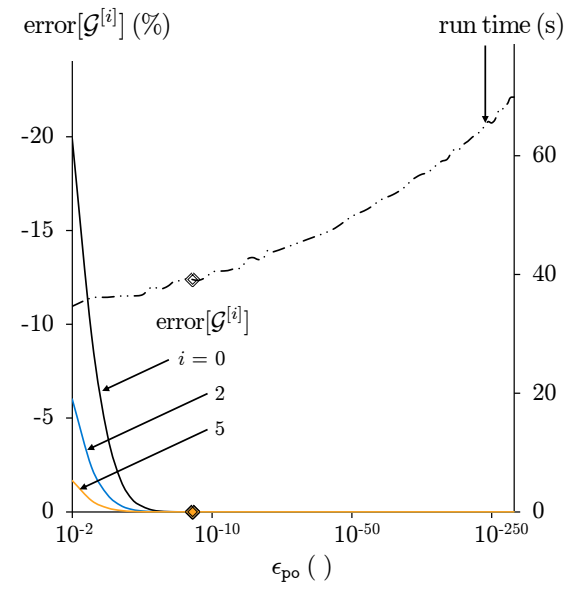

(b) Pull-out threshold.

Figure 9: Numerical convergence study (for several hierarchical levels $i$ ). Errors are relative to nominal inputs (Table 2), and run times were obtained with an Intel(R) Core(TM)2 QUAD CPU @ $2.50 \mathrm{GHz}$. Errors highlighted with $\diamond$ correspond to the nominal inputs in Table 2 .

\subsection{Parametric study}

The relation between single-fibre strength statistics and expected fracture toughnesses is shown in Figures 10 and 11. Stronger fibres lead to tougher composites (Figure 10), both in debonding and pull-out contributions. The effect of fibre strength variability (Figure 11) is more complex: as $\mathrm{CoV}_{X}^{\mathrm{f}}$ decreases from large values, so do the toughnesses of all hierarchical levels; however, this relation becomes non-monotonic for relatively low variability on fibre strength, and both components reach deterministic limits as $\mathrm{CoV}_{X}^{\mathrm{f}} \rightarrow 0$ :

$$
\left\{\begin{array}{l}
\mathcal{G}_{\mathrm{deb}}^{\left[i^{\max }\right]}\left(\mathrm{CoV}_{X}^{\mathrm{f}}=0\right)=\sum_{i=0}^{i^{\max }} \frac{C^{[i]} \cdot \mathcal{G}_{\mathrm{SL}} \cdot 2 \cdot a^{[i]}\left(X_{\mathrm{m}}^{\mathrm{f}} / k_{\mathcal{G}}^{[i]}\right)}{c_{\mathcal{G}} \cdot A^{[i]} / V^{\mathrm{f}}} \quad, \quad \text { with } a^{[i]}\left(\sigma^{\infty}\right) \text { as in Eq. 14, } \\
\mathcal{G}_{\mathrm{po}}^{\left[i^{\max }\right]}\left(\mathrm{CoV}_{X}^{\mathrm{f}}=0\right)=0
\end{array}\right.
$$

Figures 12 and 13 evaluate the effect of fibre-matrix interfacial properties on the toughness of bundles. The energy dissipated by debonding becomes negligible for very brittle interfaces; however, as the interfacial toughness increases, debonding growth is inhibited $\left(\mathcal{G}_{\mathrm{SL}} \rightarrow \infty\right.$ in Equation 14) and both toughness components progressively vanish (Figure 12). Similarly, very large frictional stresses reduce the energy dissipated by debonding $\left(\lambda^{[i]} \rightarrow \infty\right.$ in Equation 14, Figure 13a); the effect of friction on the pull-out toughness (Figure 13b) depends on the bundle level considered. 


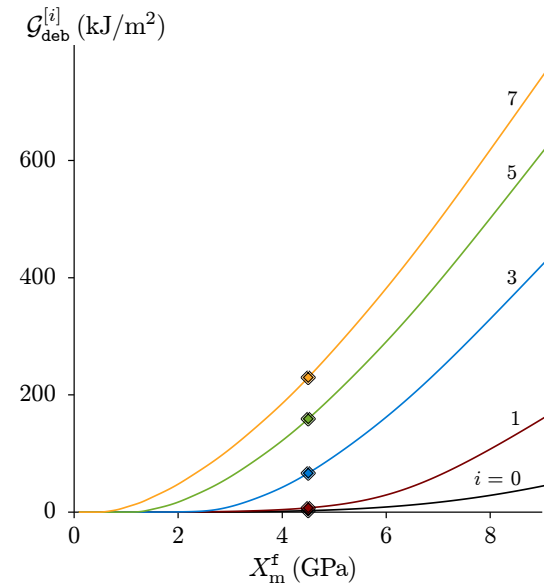

(a) Debonding component.

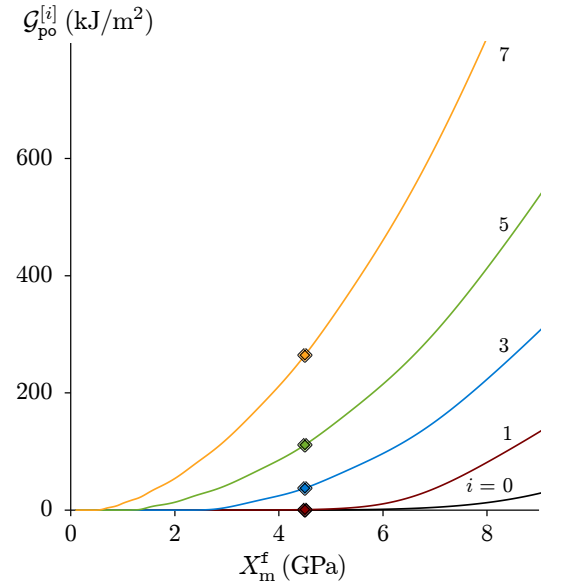

(b) Pull-out component.

Figure 10: Effect of mean fibre strength on the expected fracture toughness (for several levels $i$ ). Toughness values highlighted with $\diamond$ correspond to the nominal inputs in Table 2 .

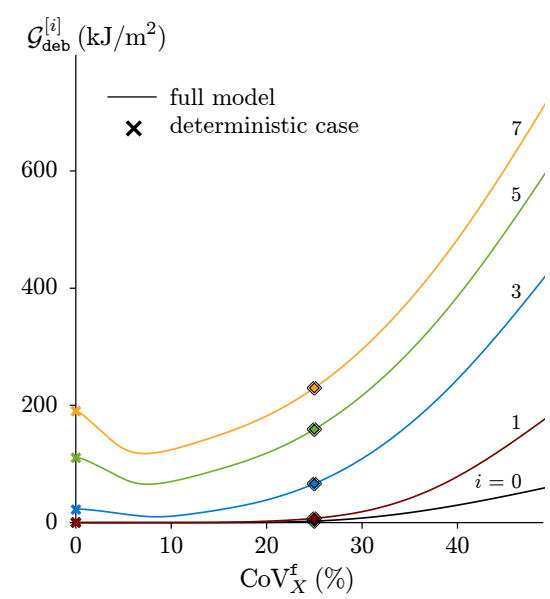

(a) Debonding component.

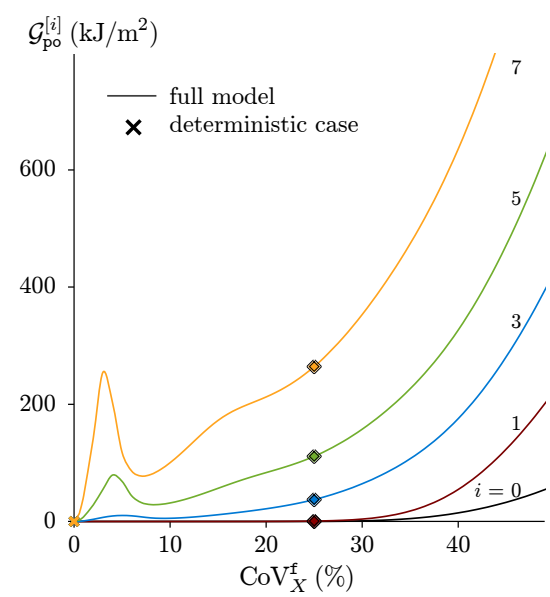

(b) Pull-out component.

Figure 11: Effect of the fibre-strength $\mathrm{CoV}$ on the expected fracture toughness (for several levels $i$ ). Toughness values highlighted with $\diamond$ correspond to the nominal inputs in Table 2 .

The influence of the assumed coordination number on the resulting fracture toughness is shown in Figure 14. For relatively large coordination numbers $\left(c_{\mathcal{G}} \gtrsim 6\right)$, such effect is very small; however, as $c_{\mathcal{G}}$ decreases to lower values, the fracture toughness is reduced as well.

\subsection{Validation against experimental results}

Figure 15 compares model predictions against literature results for the translaminar fracture toughness of UD FRPs, obtained experimentally through CT tests (Laffan 


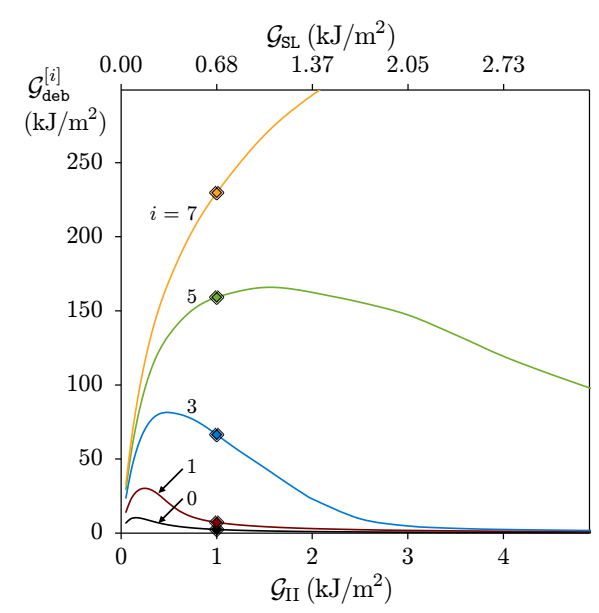

(a) Debonding component.

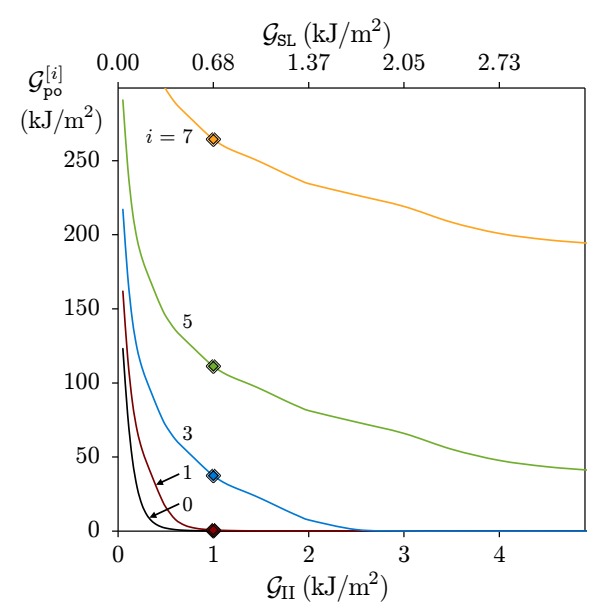

(b) Pull-out component.

Figure 12: Effect of interfacial toughness (in-situ, $\mathcal{G}_{\mathrm{SL}}$, or mode-II delamination $\mathcal{G}_{\mathrm{II}}$ ) on the expected fracture toughness (for several levels $i$ ). Toughness values highlighted with $\diamond$ correspond to the nominal inputs in Table 2 .

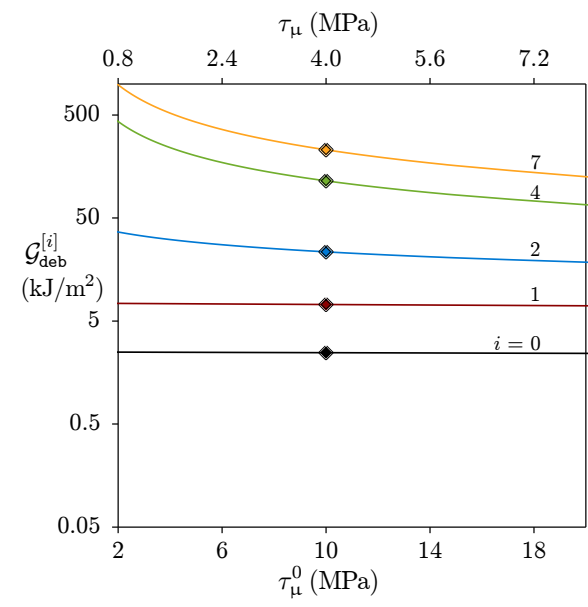

(a) Debonding component.

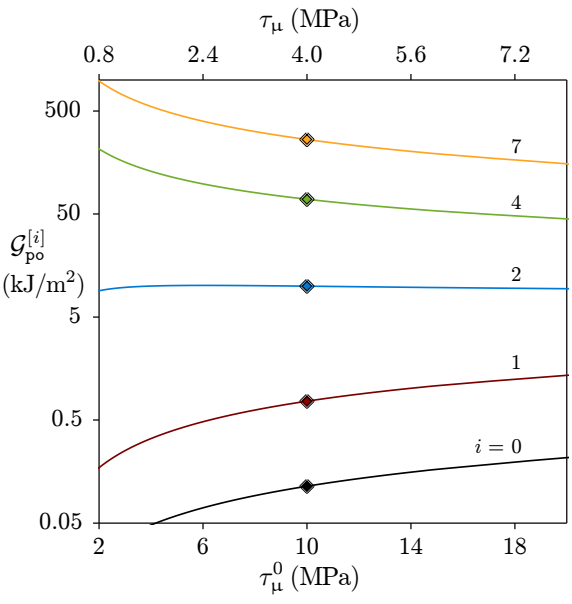

(b) Pull-out component.

Figure 13: Effect of the friction coefficient (in-situ, $\tau_{\mu}$, or in SFPO, $\tau_{\mu}^{0}$ ) on the expected fracture toughness (for several levels $i$ ). Toughness values highlighted with $\diamond$ correspond to the nominal inputs in Table 2 .

et al., 2010, Pinho et al., 2006a, Teixeira et al., 2011). The materials combine different carbon fibres, epoxy matrices and ply thicknesses (see Figure 1).

Model inputs for the validation are shown in Tables 3 to 5. Single-fibre strength parameters were determined through Single Fibre Tensile Tests (SFTT) at 10 and $20 \mathrm{~mm}$ gauge lengths (Pimenta and Pinho, 2014); the Weibull modulus $m$ was estimated using the average strengths of fibres at both gauge lengths. 


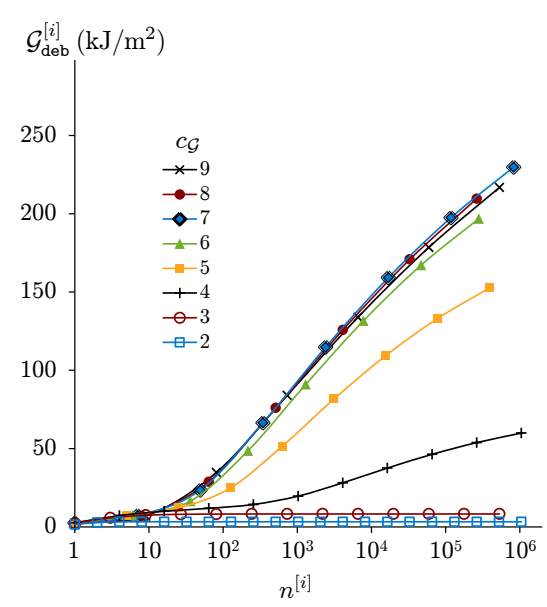

(a) Debonding component.

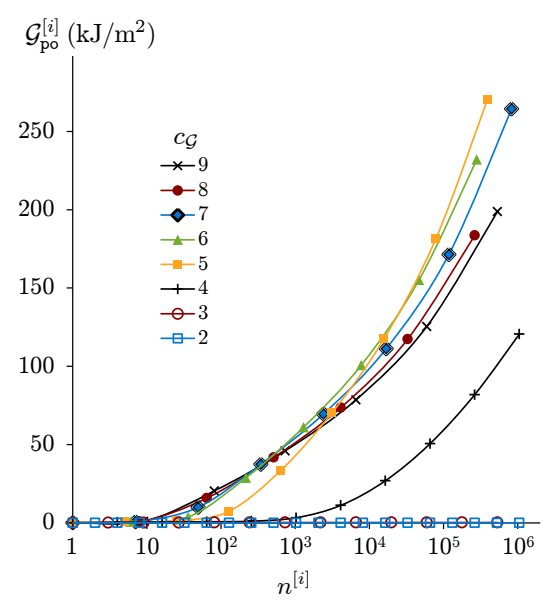

(b) Pull-out component.

Figure 14: Effect of the coordination number on the expected fracture toughness (for several levels $i$ ).

Table 3: Description of composites for model validation.

\begin{tabular}{ccccccc}
\hline $\begin{array}{c}\text { Fibre } \\
\text { type }\end{array}$ & $\begin{array}{c}\text { Epoxy } \\
\text { type }\end{array}$ & $\begin{array}{c}\text { Reference } \\
\text { for CT tests }\end{array}$ & $\begin{array}{c}\bar{X}^{\mathrm{UD}(\star)(\dagger)} \\
(\mathrm{GPa})\end{array}$ & $\begin{array}{c}\mathcal{G}_{\mathrm{II}}{ }^{(\dagger)} \\
\left(\mathrm{kJ} / \mathrm{m}^{2}\right)\end{array}$ & $\begin{array}{c}\tau_{\mu}^{0}(\S) \\
(\mathrm{MPa})\end{array}$ & $\begin{array}{c}V^{\mathrm{f}} \text { (nominal) } \\
(\%)\end{array}$ \\
\hline T300 & 913 & Pinho et al. (2006a) & 2.006 & 1.10 & 10 & 60 \\
T300 & 920 & Laffan et al. (2010) & 1.697 & 1.40 & 10 & 60 \\
T800 & M21 & Teixeira et al. (2011) & 3.067 & 1.25 & 10 & 60 \\
\hline
\end{tabular}

${ }^{(*)}$ Average strength of composite UD specimens (cross section of $1.0 \mathrm{~mm} \times 15 \mathrm{~mm}, 138 \mathrm{~mm}$ long), from Pinho et al. (2006b, T300/913), McCarroll (2008, T300/920) and Jehangir (2011, T800/M21).

( $\dagger$ Experimental mode-II delamination toughness (propagation value), from Pinho et al. (2006b, T300/913), McCarroll (2008, T300/920) and Ilyas et al. (2009, T800/M21). The corresponding in-situ debonding toughnesses (Appendix C) are $\mathcal{G}_{\mathrm{SL}}=\beta \cdot \mathcal{G}_{\mathrm{II}}$, with $\beta_{\mathrm{Q}}=0.667$ or $\beta_{\mathrm{H}}=0.683$.

(§) Typical SFPO test result (excluding Poisson's effects) for carbon-epoxy systems (Zhang et al., 1999, Zhou et al., 1992). The corresponding in-situ frictional stress at $V^{f}=60 \%$ is $\tau_{\mu}=4.0 \mathrm{MPa}$ (Appendix D).

Table 4: Fibre properties for model validation.

\begin{tabular}{cccccc}
\hline Fibre type & $\phi^{\mathrm{f}}(\star)$ & $E^{\mathrm{f}}\left({ }^{(\star)}(\mathrm{GPa})\right.$ & $\sigma_{0}^{\text {f }}{ }^{(\dagger)}(\mathrm{GPa})$ & $m^{(\dagger)}(-)$ & $l_{\mathrm{r}}(\mathrm{mm})$ \\
\hline T300 & 7.0 & 230 & 3.904 & 4.19 & 10 \\
T800 & 5.0 & 294 & 5.662 & 4.63 & 10 \\
\hline
\end{tabular}

${ }^{(\star)}$ Nominal values from Toray Carbon Fibers America, Inc (2012).

(†) Experimental SFTT result (Pimenta and Pinho, 2014), normalised for the reference length.

The strength distributions for each bundle level were calculated through the strength model developed by Pimenta and Pinho (2013); for each material system, the average strength measured in standard UD specimens $\left(\bar{X}^{\mathrm{UD}}\right)$ was used to calibrate the distribu- 
Table 5: Filament count and hierarchical level corresponding to experimental toughness measurements.

\begin{tabular}{lccccc}
\hline \multicolumn{1}{c}{ Composite } & $\begin{array}{c}t^{\mathrm{ply}} \\
(\mathrm{mm})\end{array}$ & $\begin{array}{c}\sqrt{n^{\max }} \\
(-)\end{array}$ & $\begin{array}{c}n^{\max } \\
(-)\end{array}$ & $c_{\mathcal{G}}=7(\mathrm{H})$ & $c_{\mathcal{G}}=9(\mathrm{Q})$ \\
\hline T300/913 (Pinho et al., 2006a) & 0.125 & 15.6 & 243.6 & 2.82 & 2.50 \\
T300/920 (Laffan et al., 2010) & 0.250 & 31.2 & 974.4 & 3.54 & 3.13 \\
\hline T800/M21 (Teixeira et al., 2011) & 0.125 & 21.9 & 477.5 & 3.17 & 2.81 \\
\hline
\end{tabular}

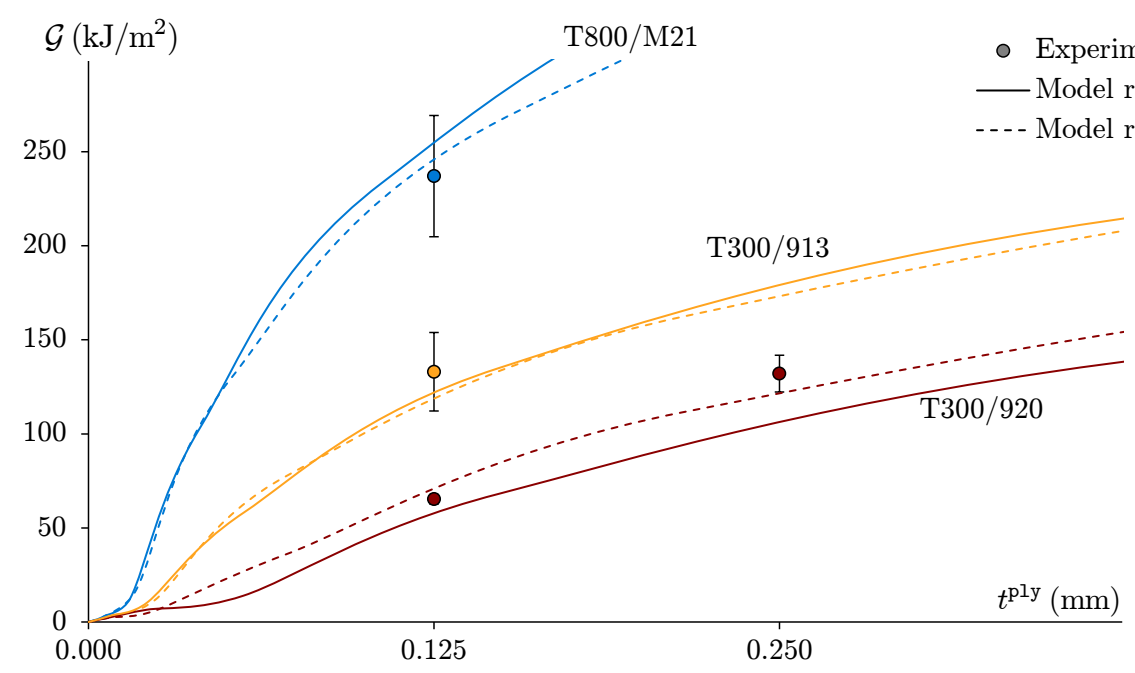

Figure 15: Validation of the fracture toughness model (for hexagonal or quadrangular configurations, see Figure 3) against experimental results (averages and standard deviations from Pinho et al. (2006a, T300/913), Laffan et al. (2010, T300/920) and Teixeira et al. (2011, T800/M21)).

tion (through the matrix/interface shear-lag strength parameter $\tau_{\mathrm{SL}}$ ).

Because the experimental results were obtained with CT specimens, it must be noted that this model does not formally account for the singularity at the notch tip, and that a global load-sharing is applies to each hierarchical level (see Section 2.2.1). It nevertheless assumes that, before the first failure event (corresponding to $i^{\max }$ ), the entire UD layer is surrounded by a partially failed level- $\left[i^{\max }+1\right]$. This generates an initial stress concentration factor $k_{\mathcal{G}}^{\left[i^{\max }\right]>1}$ and local shear, as shown in Figure 5; as failure progresses down the hierarchical levels, the stress concentration factor changes, which represents an actually heterogeneous load sharing scheme. 


\section{Discussion}

\subsection{Experimental validation}

Model results presented in Section 3 are largely supported by experimental observations on the toughness of UD composites:

a. The translaminar fracture toughness of a UD composite increases with the size of the cross section (Figure 8b and 15). This has been directly observed in CT specimens with blocked ply thickness (Laffan et al., 2010); it is also supported by a significant toughening effect observed in discontinuous recycled composites due to pull-out and failure of large bundles (Pimenta and Pinho, 2014, Pimenta et al., 2010).

b. The model predicts the formation of longer debonds and pull-outs as the number of fibres in the fracture surface grows (Figure 8d). This is corroborated by experimental observations in UD plies (Figure 1 and Laffan et al., 2010) and in discontinuous recycled composites with different degrees of bundling (Pimenta and Pinho, 2014, Pimenta et al., 2010).

c. The pull-out aspect ratios of different level- $[i]$ bundles are in the same order of magnitude for several hierarchical levels (Figure 8f); therefore, although the model imposes self-affinity only (i.e. anisotropic scaling), it actually predicts nearly selfsimilar fracture surfaces (i.e. with isotropic scaling). This is supported by experimental evidence, as shown in Figure 2.

d. The variability of pull-out lengths is larger than the variability of fibre strength (compare Figures 8a and 8f), as Wells and Beaumont (1985b) observed experimentally in carbon FRP specimens.

e. Composite toughness increases with fibre strength (Figure 10); a similar trend is suggested by experiments in carbon-epoxy systems with different fibres (Laffan et al., 2012). On the contrary, composites with very weak fibres should be brittle and present smooth fracture surfaces, as observed experimentally by Pimenta and Pinho (2012) in recycled woven composites with extreme fibre degradation.

f. Model predictions agree remarkably well with experimental fracture toughness measurements (Figure 15). While further experimental data is required for a more comprehensive validation, these results support the ability of the model to 
cope with different fibre types (T800/M21 vs. T300/913 and T300/920), different matrices (T300/913 vs. T300/920), and different ply thicknesses (T300/920 at $0.125 \mathrm{~mm}$ vs. $0.250 \mathrm{~mm}$ ).

\subsection{Interpretation of toughening mechanisms}

This model considers debonding and pull-out as the main toughnening mechanisms (Equation 27); additionally, it also accounts for fibre bridging (through $k_{\mathcal{G}}^{[i]}$ in Equation 10) and for the release of elastic energy during debonding (through $a^{[i]}\left(\sigma^{\infty}\right)$ in Equation 14). The results obtained in Section 3 provide further insight on the translaminar failure and toughening mechanisms in UD composites:

a. Each of the sequences of events for level-[i] bundle failure (Section 2.2.3) is dominant for different bundle sizes:

- Figure 8d suggests that small bundles fail through sequence $E_{2}$ : large stress concentrations $\left(k_{\mathcal{G}}^{[i]}\right.$ in Equation 10) lead to premature translaminar fracture, and the load-bearing capacity is lost as soon as a debonding propagates until the failure site (hence $\mathcal{L}_{\mathrm{deb}, \mathrm{m}}^{[i]} \approx 2 \cdot \mathcal{L}_{\mathrm{po}, \mathrm{m}}^{[i]}$ for $n^{[i]} \lesssim 20$ ).

- On the contrary, sequence $E_{1}$ prevails for large-scale bundles, which firstly debond from their neighbours (favoured by a large $A^{[i]} / C^{[i]}$ ratio, Equation 14), and then eventually fracture within the debonding length (thus $\mathcal{L}_{\mathrm{deb}, \mathrm{m}}^{[i]} \gg 2 \cdot \mathcal{L}_{\mathrm{po}, \mathrm{m}}^{[i]}$ for $n^{[i]} \gtrsim 100$ in Figure $8 \mathrm{~d}$ ).

b. For given interfacial properties, the debonding length is governed by bundle strength (Equation 14) and Figure 10a); below a given threshold (dictated by $\sigma^{\infty}=\psi^{[i]} / \kappa^{[i]}$ in Equation 14), debonding becomes negligible. This also justifies why the variability of debonding length (Figure 8e) decreases for large bundles (as does bundle strength, Figure 8a), and the similar effect of single-fibre strength variability on both $X_{\mathrm{m}}^{\left[i_{X}\right]}$ (Pimenta and Pinho, 2013) and $\mathcal{G}_{\mathrm{deb}}^{\left[i_{\mathcal{G}}\right]}$ (Figure 11a).

c. The pull-out toughness is governed by two processes:

- The debonding length defines an upper bound for the pull-out length $\left(l_{\mathrm{po}}^{[i]} \leq l_{\mathrm{deb}}^{[i]} / 2\right.$, Equation 15 and Figure 8d);

- Pull-out originates directly from the random location of defects in fibres and bundles (Figure 11b). Consequently, composites with deterministic fibre strength $\left(\mathrm{CoV}_{X}^{\mathrm{f}}=0\right)$ would present no pulled-out fibres or bundles. 
The non-monotonic relation between $\mathcal{G}_{\mathrm{po}}^{[i]}$ and $\mathrm{CoV}_{X}^{\mathrm{f}}$ (with local peak at $\left.\mathrm{CoV}_{X}^{\mathrm{f}} \approx 5-10 \%\right)$ is also very similar to that observed between $\operatorname{CoV}_{X}^{[i]}$ and $\mathrm{CoV}_{X}^{\mathrm{f}}$ in the previously developed bundle strength model (Pimenta and Pinho, 2013); this is because bundles with large strength variability are more likely to break away from the maximum stress concentration, thus dissipating more energy during pull-out.

d. The model predicts that the translaminar fracture toughness increases monotonically with the size of the fracture surface (non-negative contributions in Equation 27). The contribution of each individual level is maximised for intermediate values of $i$ (note the $g_{\mathrm{deb}}^{[i]}$ curve in Figure $8 \mathrm{c}$ and the concave-down toughness curves in Figure 15), due to competing mechanisms:

- The debonding component is directly related to the mean debonding aspect ratio $\alpha_{\mathrm{deb}}^{[i]}$ (Figure 8e). As bundle size increases, debonding is initially favoured by the decreasing ratio $C^{[i]} / A^{[i]}$ (Equation 14), but later hindered by the progressively smaller stress concentrations $\left(k_{\mathcal{G}}^{[i]}\right.$ in Equation 10).

- The pull-out component is mainly influenced by the squared mean pull-out length (Equation 25 and Figure 8f). Consequently, its contribution increases for a larger range of scales than the debonding counterpart; nevertheless, as both the debonding aspect ratio and the bundle strength variability decrease for larger bundles, $g_{\mathrm{po}}^{[i]}$ eventually falls as well.

e. The results from the parametric study suggest that the translaminar toughness of UD composites could be greatly improved by increasing mean fibre strength (Figure 10) and / or fibre strength variability (Figure 11). The effect of interfacial properties is non-monotonic (Figures 12 and 13), thus the overall toughness is maximised by intermediate values of both $\mathcal{G}_{\mathrm{SL}}$ and $\tau_{\mu}$.

\subsection{Novel model features and challenging results}

The model developed in this paper offers a novel approach to estimate the translaminar fracture toughness of UD composites, and provides some challenging results open to further validation:

a. The present model is the first attempt in the literature to combine quasi-fractal geometries with physically-based toughening mechanisms in UD composites, and 
offers the first predictions for size effects in translaminar fracture toughness. The experimental validation (Figure 15) is extremely encouraging.

b. The hierarchy in fracture surfaces is characterised by the coordination number $c_{\mathcal{G}}$, which is currently a mathematical parameter. However, $c_{\mathcal{G}}$ is likely related to physical properties of the constituent materials (e.g. fibre strength variability) and / or fracture surfaces (e.g. the fractal dimension, Carpinteri and Chiaia, 1996).

c. Figure $8 \mathrm{~b}$ suggests that the main translaminar toughening mechanism depends not only on material properties, but also on size: debonding dominates for small bundles, but pull-out becomes increasingly important as the filament count grows; at the conventional ply scale $\left(t^{\mathrm{ply}} \approx 0.125 \mathrm{~mm}, n^{[i]} \approx 2400-4800\right)$, pull-out and debonding have similar contributions. This dependence may justify the lack of agreement in the literature on which toughening mechanism dominates in CFRPs (Kim and Mai, 1991, Wells and Beaumont, 1985a).

d. The effect of interfacial properties $\left(\mathcal{G}_{\mathrm{SL}}\right.$ and $\left.\tau_{\mu}\right)$ on the overall fracture toughness (Figures 12 and 13) is complex and far from the direct proportionally suggested in Equation 1. Although maybe counter-intuitive, model predictions are supported by experimental evidence: comparing the T300/913 and T300/920 systems (Figure 15) illustrates how a tougher matrix can actually embrittle the composite, as Kim et al. (1992) observed experimentally. In addition, reducing matrix shrinkage - and, consequently, decreasing the frictional stress $\tau_{\mu}$ — has been shown to increase the translaminar toughness of CFRPs (Kim and Mai, 1991, Lim et al., 1984), corroborating the results in Figure 13 for larger bundles.

e. The model predicts that the fracture toughness increases with filament count, but reaches a plateau for very large surfaces (see Section 4.2.d.). While this agrees with the typical fractal-based scaling law (Carpinteri and Chiaia, 1996, Weiss, 2001), it cannot be experimentally validated for FRPs due to lack of data in the literature.

\section{Conclusions}

An analytical model for the translaminar fracture toughness of FRP bundles was developed, implemented and validated. The model assumes a hierarchical failure process with formation of stochastic variations of quasi-fractal fracture surfaces; debonding and 
pull-out lengths of each hierarchical level are stochastic variables, whose distribution is calculated by combining fracture mechanics with a probabilistic analysis of failure. The overall translaminar toughness includes the contributions of debonding and pull-out for all hierarchical levels formed in a fracture surface, which generates a size effect.

The model requires, as inputs, the in-situ properties of the fibre-matrix interface, as well as the bundle strength distributions associated with each hierarchical level (which can be calculated using the strength model developed by Pimenta and Pinho, 2013). It was found that the translaminar toughness is enhanced by stronger fibres with large variability, and by intermediate values of interfacial toughness and friction.

Model predictions were validated against experimentally measured translaminar fracture toughnesses. The model was able to reproduce the effect of different fibre and matrix types, as well as the marked increase of toughness for thicker plies.

This work proposes the first model for predicting size effects on the translaminar fracture toughness of FRP bundles or unidirectional plies, which is essential for the accurate simulation and design of damage-tolerant composite structures (Camanho et al., 2007, Chen et al., 2013, van der Meer et al., 2010, Pinho et al., 2006b). In addition, this model is also paramount for understanding and simulating state- of - the- $^{-}$ art multiscale discontinuous-fibre composites, both virgin (Feraboli et al., 2009, Harper et al., 2007) and recycled (Pimenta and Pinho, 2014, Pimenta et al., 2010).

\section{Acknowledgements}

The funding from the Portuguese Foundation for Science and Technology (project nr. SFRH/BD/44051/2008) is gratefully acknowledged. The authors are also thankful to Dr Matthew Laffan (Imperial College London) for providing the original micrograph shown in Figure 2b.

\section{Appendix A. Stress concentrations before level- $[i]$ failure of a quasi-fractal fracture surface}

The hierarchical failure process considered in the development of the toughness model (Figure 4 in Section 2.2) implies that, as failure progresses down the hierarchy, fibres and bundles bridging the two fracture faces feel progressively higher stress concentrations. Consider a surface immediately before level- $[i]$ failure; each surviving level-[i] bundle (with $n^{[i]}=c_{\mathcal{G}}{ }^{i}$ fibres) is surrounded by $c_{\mathcal{G}}-1$ partially broken ones; each of the latter contains a level- $[i-1]$ surviving bundle (with $n^{[i-1]}=c_{\mathcal{G}}{ }^{i-1}$ fibres), 
and $c_{\mathcal{G}}-1$ partially broken ones. Repeating this recursively down to level [0] (which then contributes with $\left(c_{\mathcal{G}}-1\right)^{i}$ bridging fibres), the fraction of surviving fibres is:

$$
\frac{1 \cdot c_{\mathcal{G}}{ }^{i}+\left(c_{\mathcal{G}}-1\right) \cdot c_{\mathcal{G}}{ }^{i-1}+\left(c_{\mathcal{G}}-1\right)^{2} \cdot c_{\mathcal{G}}{ }^{i-2}+\cdots+\left(c_{\mathcal{G}}-1\right)^{i} \cdot 1}{c_{\mathcal{G}}{ }^{i+1}}=\frac{1}{c_{\mathcal{G}}} \cdot \sum_{j=0}^{i}\left(\frac{c_{\mathcal{G}}-1}{c_{\mathcal{G}}}\right)^{j}
$$

The sum in the right hand side of Equation A.1 represents a geometric series with ratio $r=\left(c_{\mathcal{G}}-1\right) / c_{\mathcal{G}}$, and sum equal to $\Sigma=\left(1-r^{i+1}\right) /(1-r)$. As the remote load is equally shared by all surviving fibres, the stress concentration factor seen by a level- $[i]$ bundle prior to its failure is:

$$
k_{\mathcal{G}}^{[i]}=\frac{1}{\frac{1}{c_{\mathcal{G}}} \cdot \Sigma} \quad \Longrightarrow \quad k_{\mathcal{G}}^{[i]}=\frac{1}{1-\left(1-\frac{1}{c_{\mathcal{G}}}\right)^{i+1}}
$$

\section{Appendix B. Equilibrium crack length before level- $[i]$ failure of a quasi- fractal fracture surface}

The debonding distance of a level- $[i]$ bridging bundle under the remote stress $\sigma^{\infty}$ (Figure 5 in Section 2.2.2) can be calculated through fracture mechanics. Let $U$ be the internal energy, $W^{\infty}$ the work of the remote load $P, W_{\mu}$ the work done by friction forces, and $\mathcal{G}_{\mathrm{SL}}$ the debonding fracture toughness. Under load control, the equilibrium debonding distance $a^{[i]}$ verifies:

$$
G=\mathcal{G}_{\mathrm{SL}}, \quad \text { where the energy release rate is } G=\frac{1}{C^{[i]}}\left[-\frac{\mathrm{d} U}{\mathrm{~d} a^{[i]}}+\frac{\mathrm{d} W^{\infty}}{\mathrm{d} a^{[i]}}+\frac{\mathrm{d} W_{\mu}}{\mathrm{d} a^{[i]}}\right] \text {. }
$$

Neglecting the contribution of matrix deformation, the internal energy of the linearelastic fibres (modulus $E^{\mathrm{f}}$ ) in the (half-) domain $x \in[0, \ell]$ is:

$$
U=\frac{A^{[i]}}{2 \cdot E^{\mathrm{f}}} \cdot \int_{x=0}^{\ell}\left[\sigma^{\mathcal{A}}(x)\right]^{2}+\left(c_{\mathcal{G}}-1\right) \cdot\left[\sigma^{\mathcal{B}}(x)\right]^{2} \mathrm{~d} x
$$

Replacing the stress fields as in Equation 12, differentiating and re-arranging,

$$
\frac{\mathrm{d} U}{\mathrm{~d} a^{[i]}}=\frac{c_{\mathcal{G}} \cdot A^{[i]}}{2 \cdot\left(c_{\mathcal{G}}-1\right) \cdot E^{\mathrm{f}}} \cdot\left[\left(k_{\mathcal{G}}^{[i]}-1\right) \cdot \sigma^{\infty}-\lambda^{[i]} \cdot a\right]^{2}
$$

The work contribution from the remote load $P$ (with remote displacement $u^{\mathcal{A}}(\ell)$ ) is: 


$$
\frac{\mathrm{d} W^{\infty}}{\mathrm{d} a^{[i]}}=P \cdot \frac{\mathrm{d} u^{\mathcal{A}}(\ell)}{\mathrm{d} a^{[i]}}, \quad \text { where } \quad P=c_{\mathcal{G}} \cdot A^{[i]} \cdot \sigma^{\infty} \quad \text { and } \quad u^{\mathcal{A}}(\ell)=\frac{1}{E^{\mathrm{f}}} \cdot \int_{x=0}^{\ell} \sigma^{\mathcal{A}}(x) \mathrm{d} x .
$$

Simplifying,

$$
\frac{\mathrm{d} W^{\infty}}{\mathrm{d} a a^{[i]}}=\frac{c_{\mathcal{G}} \cdot A^{[i]}}{E^{f}} \cdot \sigma^{\infty} \cdot\left[\left(k_{\mathcal{G}}^{[i]}-1\right) \cdot \sigma^{\infty}-\lambda^{[i]} \cdot a^{[i]}\right] .
$$

The energy dissipated by the constant frictional stresses $\tau_{\mu}$ is:

$$
W_{\mu}=-\tau_{\mu} \cdot C^{[i]} \cdot \int_{x=0}^{a^{[i]}} \llbracket u(x) \rrbracket \mathrm{d} x,
$$

where $\tau_{\mu} \cdot C^{[i]}=\lambda^{[i]} \cdot A^{[i]}$ (Equation 13) and the displacement jump is:

$$
\llbracket u(x) \rrbracket=u^{\mathcal{B}}(x)-u^{\mathcal{A}}(x)=\frac{1}{E^{\mathrm{f}}} \cdot \int_{\xi=x}^{a^{[i]}} \sigma^{\mathcal{A}}(\xi)-\sigma^{\mathcal{B}}(\xi) \mathrm{d} \xi .
$$

Using the stress field in Equation 12,

$$
\llbracket u(x) \rrbracket=\frac{c_{\mathcal{G}} \cdot\left(x-a^{[i]}\right)}{\left(c_{\mathcal{G}}-1\right) \cdot E^{\mathrm{f}}} \cdot\left[\left(k_{\mathcal{G}}^{[i]}-1\right) \cdot \sigma^{\infty}-\frac{1}{2} \cdot \lambda^{[i]} \cdot\left(x+a^{[i]}\right)\right],
$$

and thus differentiating Equation B.6 yields:

$$
\frac{\mathrm{d} W_{\mu}}{\mathrm{d} a^{[i]}}=-\frac{c_{\mathcal{G}} \cdot A^{[i]}}{\left(c_{\mathcal{G}}-1\right) \cdot E^{\mathrm{f}}} \cdot \lambda^{[i]} \cdot a^{[i]} \cdot\left[\left(k_{\mathcal{G}}^{[i]}-1\right) \cdot \sigma^{\infty}-\lambda^{[i]} \cdot a^{[i]}\right] .
$$

Replacing Equations B.3, B.5 and B.9 in Equation B.1 and simplifying,

$G=\mathcal{G}_{\mathrm{SL}} \Longrightarrow \frac{c_{\mathcal{G}} \cdot A^{[i]}}{2 \cdot E^{\mathrm{f}} \cdot C^{[i]}} \cdot \frac{\left(2 \cdot c_{\mathcal{G}}-k_{\mathcal{G}}^{[i]}-1\right) \cdot \sigma^{\infty}-\lambda^{[i]} \cdot a^{[i]}}{c_{\mathcal{G}}-1} \cdot\left[\left(k_{\mathcal{G}}^{[i]}-1\right) \cdot \sigma^{\infty}-\lambda^{[i]} \cdot a^{[i]}\right]=\mathcal{G}_{\mathrm{SL}}$

which defines the equilibrium crack length.

Additionally, one must also impose that $G^{\prime}\left(a^{[i]}\right) \leq \mathcal{G}_{\mathrm{SL}}{ }^{\prime}\left(a^{[i]}\right)$ to ensure stable crack propagation. According to the quadratic relation between $G$ and $a^{[i]}$ defined above, $G^{\prime}\left(a^{[i]}\right) \leq 0$ for the smallest solution of Equation B.10. The debonding toughness is assumed to be independent of crack length, hence $\mathcal{G}_{\mathrm{SL}}^{\prime}\left(a^{[i]}\right)=0$ (see Appendix $\mathrm{C}$ for further details). The stability condition is therefore fulfilled by taking the smallest root of Equation B.10, which defines the the equilibrium crack length at the remote stress 
$\sigma^{\infty}$ as:

$$
a^{[i]}\left(\sigma^{\infty}\right)=\frac{1}{\lambda^{[i]}} \cdot\left[\left(c_{\mathcal{G}}-1\right) \cdot \sigma^{\infty}-\sqrt{\left[\left(c_{\mathcal{G}}-k_{\mathcal{G}}^{[i]}\right) \cdot \sigma^{\infty}\right]^{2}+\frac{2 \cdot\left(c_{\mathcal{G}}-1\right) \cdot E^{\mathrm{f}} \cdot \mathcal{G}_{\mathrm{SL}} \cdot C^{[i]}}{c_{\mathcal{G}} \cdot A^{[i]}}}\right]
$$

\section{Appendix C. Mode-II toughness for debonding}

The dissipation mechanisms associated with debonding between a fibre or bundle and the surrounding material (in-situ mode-II toughness, $\mathcal{G}_{\mathrm{SL}}$ ) are similar to those contributing to the mode-II delamination toughness $\left(\mathcal{G}_{\mathrm{II}}\right.$, measured for instance through End Notched Flexure (ENF) tests). However, while the former considers an idealised microscopic texture of the debonded surface (see Figure 3), the latter is usually normalised by the macroscopic (smoothed) delaminated area. The toughnesses $\mathcal{G}_{\mathrm{SL}}$ and $\mathcal{G}_{\mathrm{II}}$ are thus related by the respective perimeters $C_{\text {micro }}$ and $C_{\text {macro }}$ :

$$
\mathcal{G}_{\mathrm{SL}}=\beta \cdot \mathcal{G}_{\mathrm{II}} \quad \text { where } \quad \beta=\frac{C_{\text {macro }}}{C_{\text {micro }}}
$$

Considering that debonding follows preferentially the fibre-matrix interface (Figure 3), the factor $\beta$ (depending on fibre arrangement) is:

$$
\begin{aligned}
& \beta_{\mathrm{Q}}=\frac{s_{\mathrm{Q}}+\phi^{\mathrm{f}}}{s_{\mathrm{Q}}+\frac{\pi}{2} \cdot \phi^{\mathrm{f}}} \stackrel{\text { Eq. }}{=} \frac{1}{1-2 \cdot \sqrt{\frac{V^{\mathrm{f}}}{\pi}+\sqrt{V^{\mathrm{f}} \cdot \pi}}}, \\
& \beta_{\mathrm{H}}=\frac{s_{\mathrm{H}}+\phi^{\mathrm{f}}}{s_{\mathrm{H}}+\frac{\pi}{2} \cdot \phi^{\mathrm{f}}} \stackrel{\text { Eq. }}{=} \frac{1}{1-\sqrt{\frac{2 \cdot \sqrt{3} \cdot V^{\mathrm{f}}}{\pi}}+\sqrt{\frac{\sqrt{3} \cdot V^{\mathrm{f}} \cdot \pi}{2}}} .
\end{aligned}
$$

Because $C_{\text {macro }} \leq C_{\text {micro }}$, the in-situ toughness $\mathcal{G}_{\mathrm{SL}}$ is smaller than the macroscopic $\mathcal{G}_{\mathrm{II}}$.

While some standard tests for measuring mode-II delamination toughness (e.g. ENF) present a rising $\mathrm{R}$-curve, this model considers $\mathcal{G}_{\mathrm{SL}}$ to be independent of the debonding length $a^{[i]}$. In order to include the total amount of energy dissipated by debonding, the propagation value of $\mathcal{G}_{\text {II }}$ (if available) should be preferred over the initiation value. 

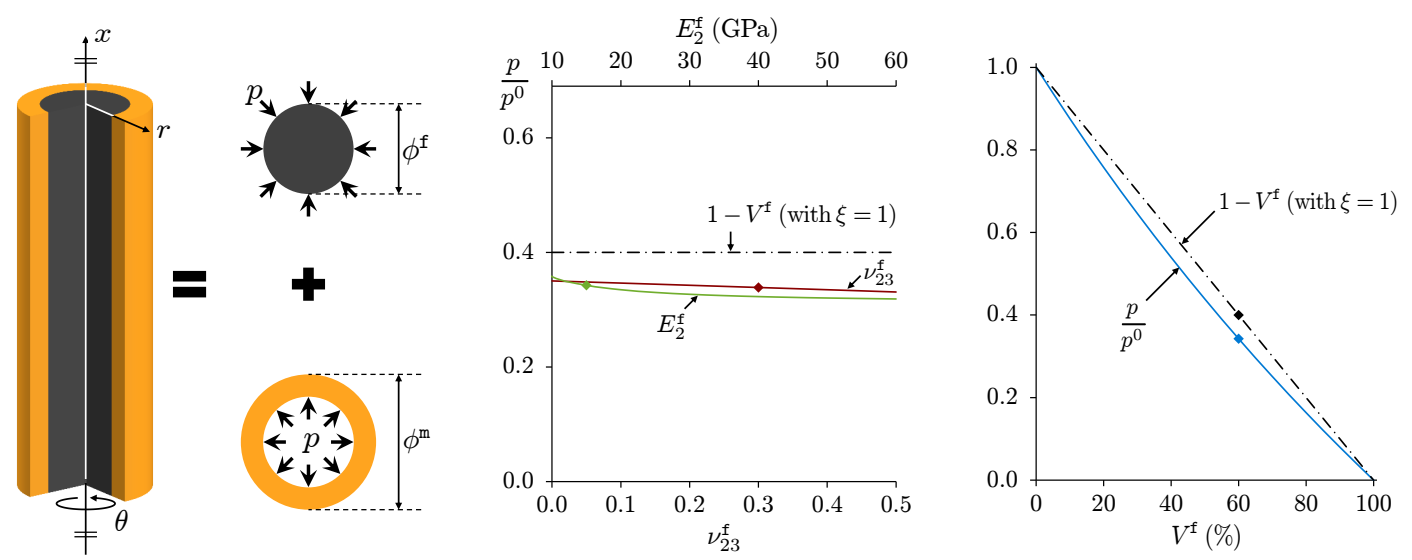

(a) Two-cylinders model for in- (b) Sensitivity of $p / p^{0}$ to fibre (c) Full model $\left(p / p^{0}\right.$ in terfacial pressure.

transverse properties.

Eq. D.6) vs. simplified $p / p^{0} \approx 1-V^{\mathrm{f}}$.

Figure D.1: Model for determination of in-situ frictional stresses. Nominal inputs are $E^{\mathrm{m}}=4 \mathrm{GPa}$, $\nu^{\mathrm{m}}=0.35, E_{2}^{\mathrm{f}}=15 \mathrm{GPa}, \nu_{23}^{\mathrm{f}}=0.20$ and $V^{\mathrm{f}}=60 \%$; nominal outputs are highlighted as $\diamond$.

\section{Appendix D. Frictional stresses during pull-out}

Considering Coulomb friction, the in-situ pull-out frictional stress $\left(\tau_{\mu}\right)$ is

$$
\tau_{\mu}=p \cdot \mu,
$$

where $p$ is the fibre-matrix interfacial compressive stress and $\mu$ is the friction coefficient. Frictional stresses are usually measured in model composites, with an extremely high matrix content (e.g. SFPO tests). In this case, matrix contraction around the fibre is not representative of that in a real composite (with $V^{\mathrm{f}} \approx 60 \%$ ), hence the need to find a relation between the frictional stresses in both cases.

Consider the two-cylinders model in Figure D.1a, representing a fibre surrounded by a matrix ring (with external diameter $\phi^{\mathrm{m}}=\phi^{\mathrm{f}} / \sqrt{V^{\mathrm{f}}}$ ). The curing process induces stress-free deformations represented by $\varepsilon_{\Delta T}^{\mathrm{f}}$ and $\varepsilon_{\Delta T}^{\mathrm{m}}$; these are different due to the mismatch of thermal expansion coefficients in the two constituents, and to the chemical contraction of the matrix.

This mismatch generates elastic stresses in the system to ensure compatibility of radial deformation between fibres and matrix at their interface, represented by $\varepsilon_{\theta}\left(\phi^{\mathrm{f}} / 2\right)$. Considering only radial $(r)$ and circumferential $(\theta)$ stresses, a linear thermo-elastic 
analysis yields:

$$
\begin{gathered}
\varepsilon_{\theta}^{\mathrm{f}}\left(\phi^{\mathrm{f}} / 2\right)=\varepsilon_{\theta}^{\mathrm{m}}\left(\phi^{\mathrm{f}} / 2\right) \Rightarrow \\
\Rightarrow \varepsilon_{\Delta T}^{\mathrm{f}}+\frac{\sigma_{\theta}^{\mathrm{f}}\left(\phi^{\mathrm{f}} / 2\right)}{E_{2}^{\mathrm{f}}}-\nu_{23}^{\mathrm{f}} \cdot \frac{\sigma_{\mathrm{r}}^{\mathrm{f}}\left(\phi^{\mathrm{f}} / 2\right)}{E_{2}^{\mathrm{f}}}=\varepsilon_{\Delta T}^{\mathrm{m}}+\frac{\sigma_{\theta}^{\mathrm{m}}\left(\phi^{\mathrm{f}} / 2\right)}{E^{\mathrm{m}}}-\nu^{\mathrm{m}} \cdot \frac{\sigma_{\mathrm{r}}^{\mathrm{m}}\left(\phi^{\mathrm{f}} / 2\right)}{E^{\mathrm{m}}} .
\end{gathered}
$$

Here the matrix is isotropic (Young's modulus $E^{\mathrm{m}}$, Poisson's ratio $\nu^{\mathrm{m}}$ ), while the fibre is transversely orthotropic (transverse elastic modulus $E_{2}^{\mathrm{f}}$ and Poisson's ratio $\nu_{23}^{\mathrm{f}}$ ). Equation D.2 neglects Poisson's effects due to axial loading; while this could be easily incorporated in the SFPO analysis (Gao et al., 1988, Zhang et al., 1999, Zhou et al., 1992), the resulting non-linear stress profiles would impede the calculation of bundle survival probabilities according to Appendix E.

Radial $\left(\sigma_{\mathrm{r}}\right)$ and circumferential $\left(\sigma_{\theta}\right)$ stresses are related by Lamé's equations for thick-walled cylinders of internal radius $r_{\mathrm{i}}$ and external radius $r_{\mathrm{e}}$, under internal $\left(p_{\mathrm{i}}\right)$ and external $\left(p_{\mathrm{e}}\right)$ pressure,

$$
\left\{\begin{array} { l } 
{ \sigma _ { \mathrm { r } } ( r ) = M _ { 0 } + M _ { 1 } \cdot \frac { 1 } { r ^ { 2 } } } \\
{ \sigma _ { \theta } ( r ) = M _ { 0 } - M _ { 1 } \cdot \frac { 1 } { r ^ { 2 } } }
\end{array} \quad \text { with } \quad \left\{\begin{array}{l}
M_{0}=\frac{p_{\mathrm{i}} \cdot r_{\mathrm{i}}^{2}-p_{\mathrm{e}} \cdot r_{\mathrm{e}}^{2}}{r_{\mathrm{e}}^{2}-r_{\mathrm{i}}^{2}} \\
M_{1}=\frac{r_{\mathrm{i}}^{2} \cdot r_{\mathrm{e}}^{2} \cdot\left(p_{\mathrm{e}}-p_{\mathrm{i}}\right)}{r_{\mathrm{e}}^{2}-r_{\mathrm{i}}^{2}}
\end{array}\right.\right.
$$

The boundary conditions are represented in Figure D.1a. The interference pressure at the fibre-matrix interface is $p$, and no external pressure is applied to the matrix ring. This is valid both for the SFPO test (with $\phi^{\mathrm{m}} \gg \phi^{\mathrm{f}}$ ), and as an axi-symmetric representative cell of a composite with the specified $V^{f}$ (as the fibre-matrix cylinder must be in equilibrium with the homogenised surrounding). Applying Equation D.3 to the model in Figure D.1a yields:

$$
\sigma_{\mathrm{r}}^{\mathrm{f}}\left(\phi^{\mathrm{f}} / 2\right)=\sigma_{\theta}^{\mathrm{f}}\left(\phi^{\mathrm{f}} / 2\right)=\sigma_{\mathrm{r}}^{\mathrm{m}}\left(\phi^{\mathrm{f}} / 2\right)=-p \quad, \quad \sigma_{\theta}^{\mathrm{m}}\left(\phi^{\mathrm{f}} / 2\right)=p \cdot \frac{1+V^{\mathrm{f}}}{1-V^{\mathrm{f}}}
$$

Replacing these in Equation D.2 and solving to $p$ results in:

$$
p=\frac{\left(-\varepsilon_{\Delta T}^{\mathrm{m}}+\varepsilon_{\Delta T}\right) \cdot E_{2}^{\mathrm{f}} \cdot E^{\mathrm{m}} \cdot\left(1-V^{\mathrm{f}}\right)}{E_{2}^{\mathrm{f}} \cdot\left(1+\nu^{\mathrm{m}}\right)+E^{\mathrm{m}} \cdot\left(1-\nu_{23}^{\mathrm{f}}\right)+V^{\mathrm{f}} \cdot\left[E_{2}^{\mathrm{f}} \cdot\left(1-\nu^{\mathrm{m}}\right)-E^{\mathrm{m}} \cdot\left(1-\nu_{23}^{\mathrm{f}}\right)\right]} .
$$

In a SFPO test, $V^{f} \rightarrow 0$; let the interfacial pressure in this case be represented as $p_{0}$. The relation between the latter and the interfacial pressure in a generic composite 
with fibre content $V^{\mathrm{f}}$ is:

$$
\begin{aligned}
& p=\xi \cdot\left(1-V^{\mathrm{f}}\right) \cdot p_{0} \quad, \text { where } \\
& \quad \xi=\frac{E_{2}^{\mathrm{f}} \cdot\left(1+\nu^{\mathrm{m}}\right)+E^{\mathrm{m}} \cdot\left(1-\nu_{23}^{\mathrm{f}}\right)}{E_{2}^{\mathrm{f}} \cdot\left(1+\nu^{\mathrm{m}}\right)+E^{\mathrm{m}} \cdot\left(1-\nu_{23}^{\mathrm{f}}\right)+V^{\mathrm{f}} \cdot\left[E_{2}^{\mathrm{f}} \cdot\left(1-\nu^{\mathrm{m}}\right)-E^{\mathrm{m}} \cdot\left(1-\nu_{23}^{\mathrm{f}}\right)\right]}
\end{aligned}
$$

For reasonable values of matrix properties, $\xi$ is nearly independent of $E_{2}^{\mathrm{f}}$ and $\nu_{23}^{\mathrm{f}}$ (Figure D.1b); this is particularly important because transverse fibre properties are extremely difficult to measure. Moreover, $\xi \approx 1$ is a sensible approximation that greatly reduces the number of required inputs (Figure D.1c).

Following Equations D.1 and D.6, and if $\tau_{\mu}^{0}$ is measured during SFPO, the frictional stresses during pull-out can be therefore approximated by:

$$
\tau_{\mu}=\xi \cdot\left(1-V^{\mathrm{f}}\right) \cdot \tau_{\mu}^{0} \approx\left(1-V^{\mathrm{f}}\right) \cdot \tau_{\mu}^{0}
$$

\section{Appendix E. Survival probability for a bundle under triangular stress field with frictional shear-lag}

Consider a level-[i] bundle of length $l_{\mathrm{r}}$ under uniform tensile stresses $\sigma$, with corresponding survival probability $S_{\mathrm{U}, \mathrm{r}}^{[i]}(\sigma)$. Assume that the bundle is now under a linear stress field (subscript L) from $\sigma=0$ to $\sigma=\sigma^{\max }$; extending the Weakest Link Theory (WLT) to non-uniform stress fields (Pimenta and Pinho, 2013), the bundle survival probability is now $S_{\mathrm{L}, \mathrm{r}}^{[i]}\left(\sigma^{\max }\right)$ such that:

$$
\ln \left[S_{\mathrm{L}, \mathrm{r}}^{[i]}\left(\sigma^{\max }\right)\right]=\frac{1}{\sigma^{\max }} \cdot \int_{\sigma=0}^{\sigma^{\max }} \ln \left[S_{\mathrm{U}, \mathrm{r}}^{[i]}(\sigma)\right] \mathrm{d} \sigma
$$

The triangular stress field shown in Figure $6 \mathrm{c}$ is equivalent to a linear stress field with length $l_{\lambda}^{[i]}=2 \cdot \sigma^{\max } / \lambda^{[i]}$. According to the WLT, the corresponding survival probability for a level-[i] bundle, $S_{\lambda}^{[i]}\left(\sigma^{\max }\right)$, verifies

$$
\ln \left[S_{\lambda}^{[i]}\left(\sigma^{\max }\right)\right]=\frac{l_{\lambda}^{[i]}}{l_{\mathrm{r}}} \cdot \ln \left[S_{\mathrm{L}, \mathrm{r}}^{[i]}\left(\sigma^{\max }\right)\right]=\frac{2}{\lambda^{[i] \cdot} \cdot l_{\mathrm{r}}} \cdot \int_{\sigma=0}^{\sigma^{\max }} \ln \left[S_{\mathrm{U}, \mathrm{r}}^{[i]}(\sigma)\right] \mathrm{d} \sigma .
$$

The equations above are used in Section 2.3 to calculate debonding and pull-out length distributions. They are valid for linear bundle stresses during progressive failure (as shown in Figure 5 and Equation 12), which neglects Poisson's effects. Including the latter in the analysis (considering for instance the stress profiles derived by Gao et al., 
1988, Wells and Beaumont, 1982) would make in-situ bundle survival probabilities much more complex to calculate, hence the use of a simplified formulation.

\section{Appendix F. Derivation of the level- $[i]$ pull-out length distribution in a quasi-fractal fracture surface}

Consider the pull-out length cCDF $S_{\mathrm{po}}^{[i]}\left(l_{\mathrm{po}}\right)$ defined in Equation 22a. Changing the integration variable from $a^{[i]}$ to $\sigma^{\infty}$ (unequivocally related in Equation 14),

$$
S_{\mathrm{po}}^{[i]}\left(l_{\mathrm{po}}\right)=\operatorname{Pr}\left(L_{\mathrm{po}}^{[i]}>l_{\mathrm{po}}^{[i]}\right)=\int_{\sigma^{\infty}=\sigma^{\min }\left(l_{\mathrm{po}}\right)}^{\infty} \operatorname{Pr}\left(X_{\mathrm{deb}}^{[i]}=\sigma^{\infty} \wedge L_{\mathrm{po}}^{[i]} \geq l_{\mathrm{po}}^{[i]}\right),
$$

where the lower integration limit verifies $a^{[i]}\left(\sigma^{\mathrm{min}}\right)=l_{\mathrm{po}}^{[i]}$; following the definition of $a^{[i]}\left(\sigma^{\infty}\right)$ in Equation 14,

$$
\sigma^{\min }\left(l_{\mathrm{po}}\right)=\frac{\lambda^{[i]} \cdot l_{\mathrm{po}}^{[i]}}{\kappa^{[i]}} \cdot\left(c_{\mathcal{G}}-1+\sqrt{\frac{\psi^{[i]} \cdot \kappa^{[i]}}{\left(\lambda^{[i]} \cdot l_{\mathrm{po}}\right)^{2}}+\left(c_{\mathcal{G}}-k_{\mathcal{G}}^{[i]}\right)^{2}}\right) .
$$

The integrand function in Equation F.1 has been defined in Equation 21 from the auxiliary distributions $S_{\mathrm{I}}^{[i]}\left(\sigma^{\infty}, l_{\mathrm{po}}^{[i]}\right)$ and $F_{\mathrm{II}}^{[i]}\left(\sigma^{\infty}, l_{\mathrm{po}}^{[i]}\right)$. Considering their representation in Figure 6, these can be calculated from $S_{\lambda}^{[i]}\left(\sigma^{\max }\right)$ (defined in Equation 17) as:

$$
S_{\mathrm{I}}^{[i]}\left(\sigma^{\infty}, l_{\mathrm{po}}^{[i]}\right)=\frac{S_{\lambda}^{[i]}\left(\sigma_{0}^{[i]}\left(\sigma^{\infty}\right)\right)}{S_{\lambda}^{[i]}\left(\sigma_{l_{\mathrm{po}}}^{[i]}\left(\sigma^{\infty}, l_{\mathrm{po}}^{[i]}\right)\right)} \quad \text { and } \quad F_{\mathrm{II}}^{[i]}\left(\sigma^{\infty}, l_{\mathrm{po}}^{[i]}\right)=1-\frac{S_{\lambda}^{[i]}\left(\sigma_{l_{\mathrm{po}}}^{[i]}\left(\sigma^{\infty}, l_{\mathrm{po}}^{[i]}\right)\right)}{S_{\lambda}^{[i]}\left(\sigma_{a}^{[i]}\left(\sigma^{\infty}\right)\right)}
$$

The differential of the latter, omitting the variables $\sigma^{\infty}$ and $l_{\mathrm{po}}^{[i]}$ in $\sigma_{l_{\mathrm{po}}}^{[i]}$ and $\sigma_{a}^{[i]}$, is:

$$
\mathrm{d} F_{\mathrm{II}}^{[i]}\left(\sigma^{\infty}, l_{\mathrm{po}}^{[i]}\right)=-\frac{1}{S_{\lambda}^{[i]}\left(\sigma_{a}^{[i]}\right)} \cdot\left(\frac{\mathrm{d}}{\mathrm{d} \sigma^{\infty}}\left[S_{\lambda}^{[i]}\left(\sigma_{l_{\mathrm{po}}}^{[i]}\right)\right]-S_{\lambda}^{[i]}\left(\sigma_{l_{\mathrm{po}}}^{[i]}\right) \cdot \frac{\frac{\mathrm{d}}{\mathrm{d} \sigma^{\infty}}\left[S_{\lambda}^{[i]}\left(\sigma_{a}^{[i]}\right)\right]}{S_{\lambda}^{[i]}\left(\sigma_{a}^{[i]}\right)}\right) \mathrm{d} \sigma^{\infty}
$$

Replacing Equations F.3 and F.4 into the definition of $\operatorname{Pr}\left(X_{\mathrm{deb}}^{[i]}=\sigma^{\infty} \wedge L_{\mathrm{po}}^{[i]} \geq l_{\mathrm{po}}^{[i]}\right)$ (Equation 21) and re-arranging yields: 
$\operatorname{Pr}\left(X_{\mathrm{deb}}^{[i]}=\sigma^{\infty} \wedge L_{\mathrm{po}}^{[i]} \geq l_{\mathrm{po}}^{[i]}\right)=-\frac{S_{\lambda}^{[i]}\left(\sigma_{0}^{[i]}\right)}{S_{\lambda}^{[i]}\left(\sigma_{a}^{[i]}\right)} \cdot\left(\frac{\frac{\mathrm{d}}{\mathrm{d} \sigma^{\infty}}\left[S_{\lambda}^{[i]}\left(\sigma_{l_{\mathrm{po}}}^{[i]}\right)\right]}{S_{\lambda}^{[i]}\left(\sigma_{l_{\mathrm{po}}}^{[i]}\right)}-\frac{\frac{\mathrm{d}}{\mathrm{d} \sigma^{\infty}}\left[S_{\lambda}^{[i]}\left(\sigma_{a}^{[i]}\right)\right]}{S_{\lambda}^{[i]}\left(\sigma_{a}^{[i]}\right)}\right) \mathrm{d} \sigma^{\infty}$

for all $\sigma^{\infty}>\sigma^{\text {min }}$. The first factor in Equation F.5 is $S_{\lambda}^{[i]}\left(\sigma_{0}^{[i]}\right) / S_{\lambda}^{[i]}\left(\sigma_{a}^{[i]}\right)=S_{\text {deb }}^{[i]}\left(\sigma^{\infty}\right)$ (Equation 18). The derivative terms can be calculated by applying the chain and logarithmic differentiation rules, and the definition of $S_{\lambda}^{[i]}\left(\sigma^{\max }\right)$ :

$$
\frac{\frac{\mathrm{d}}{\mathrm{d} \sigma^{\infty}}\left[S_{\lambda}^{[i]}\left(\sigma^{\max }\right)\right]}{S_{\lambda}^{[i]}\left(\sigma^{\max }\right)}=\frac{\mathrm{d} \sigma^{\max }}{\mathrm{d} \sigma^{\infty}} \cdot \frac{\mathrm{d}}{\mathrm{d} \sigma^{\max }} \ln \left[S_{\lambda}^{[i]}\left(\sigma^{\max }\right)\right] \stackrel{\text { Eq. }}{=}{ }^{17} \frac{\mathrm{d} \sigma^{\max }}{\mathrm{d} \sigma^{\infty}} \cdot \frac{2}{\lambda^{[i]} \cdot l_{\mathrm{r}}} \cdot \ln \left[S_{\mathrm{U}, \mathrm{r}}^{[i]}\left(\sigma^{\max }\right)\right]
$$

Equation F.5 then simplifies to:

$$
\begin{aligned}
& \operatorname{Pr}\left(X_{\mathrm{deb}}^{[i]}=\sigma^{\infty} \wedge L_{\mathrm{po}}^{[i]} \geq l_{\mathrm{po}}^{[i]}\right)= \\
& \quad-S_{\mathrm{deb}}^{[i]}\left(\sigma^{\infty}\right) \cdot \frac{2}{l_{\mathrm{r}} \cdot \lambda^{[i]}} \cdot\left(\frac{\mathrm{d} \sigma_{l_{\mathrm{po}}}^{[i]}}{\mathrm{d} \sigma^{\infty}} \cdot \ln \left[S_{\mathrm{U}, \mathrm{r}}^{[i]}\left(\sigma_{l_{\mathrm{po}}}^{[i]}\right)\right]-\frac{\mathrm{d} \sigma_{a}^{[i]}}{\mathrm{d} \sigma^{\infty}} \cdot \ln \left[S_{\mathrm{U}, \mathrm{r}}^{[i]}\left(\sigma_{a}^{[i]}\right)\right]\right) \mathrm{d} \sigma^{\infty},
\end{aligned}
$$

where, following the definition of $\sigma_{a}^{[i]}\left(\sigma^{\infty}\right)$ and $\sigma_{l_{\mathrm{po}}}^{[i]}\left(\sigma^{\infty}\right)$ in Equations 19 and 20,

$$
\frac{\mathrm{d} \sigma_{a}^{[i]}}{\mathrm{d} \sigma^{\infty}}=1-c_{\mathcal{G}}+k_{\mathcal{G}}^{[i]}+\frac{\left(c_{\mathcal{G}}-k_{\mathcal{G}}^{[i]}\right)^{2} \cdot \sigma^{\infty}}{\sqrt{\left[\left(c_{\mathcal{G}}-k_{\mathcal{G}}^{[i]}\right) \cdot \sigma^{\infty}\right]^{2}+\psi^{[i]}}} \quad \text { and } \quad \frac{\mathrm{d} \sigma_{l_{\mathrm{po}}}^{[i]}}{\mathrm{d} \sigma^{\infty}}=k_{\mathcal{G}}^{[i]}
$$

Integrating Equation F.7 for $\sigma^{\infty} \geq \sigma^{\text {min }}$ finally leads to the pull-out cCDF presented in Equation 22b.

\section{References}

Balankin, A.S., 1997. Physics of fracture and mechanics of self-affine cracks. Engineering Fracture Mechanics 57, 135-203.

Bažant, Z.P., 1997. Scaling of quasibrittle fracture: hypotheses of invasive and lacunar fractality, their critique and weibull connection. International Journal of Fracture 83, $41-65$.

Bažant, Z.P., Kazemi, M.T., 1990. Determination of fracture energy, process zone 
length and brittleness number from size effect, with application to rock and concrete. International Journal of Fracture 44, 111-131.

Bažant, Z.P., Yavari, A., 2005. Is the cause of size effect on structural strength fractal or energetic-statistical? Engineering Fracture Mechanics 72, 1-31.

Beyerlein, I.J., Phoenix, S.L., 1996. Stress concentrations around multiple fiber breaks in an elastic matrix with local yielding or debonding using quadratic influence superposition. Journal of the Mechanics and Physics of Solids 44, 1997-2039.

Beyerlein, I.J., Phoenix, S.L., 1997a. Statistics of fracture for an elastic notched composite lamina containing Weibull fibers - Part I. Features from Monte-Carlo Simulation. Engineering Fracture Mechanics 57, 241-265.

Beyerlein, I.J., Phoenix, S.L., 1997b. Statistics of fracture for an elastic notched composite lamina containing Weibull fibers - Part II. Probability models of crack growth. Engineering Fracture Mechanics 57, 267-299.

Borodich, F.M., 1997. Some fractal models of fracture. Journal of the Mechanics and Physics of Solids 45, 239-259.

Camanho, P.P., Maimi, P., Dávila, C.G., 2007. Prediction of size effects in notched laminates using continuum damage mechanics. Composites Science and Technology 67, 2715-2727.

Carpinteri, A., 1994. Scaling laws and renormalization-groups for strength and toughness of disordered materials. International Journal of Solids and Structures 31, 291302.

Carpinteri, A., Chiaia, B., 1995. Multifractal nature of concrete fracture surfaces and size effects on nominal fracture energy. Materials and Structures 28, 435-443.

Carpinteri, A., Chiaia, B., 1996. Crack-resistance behavior as a consequence of selfsimilar fracture topologies. International Journal of Fracture 76, 327-340.

Carpinteri, A., Paggi, M., 2009. A top-down approach for the prediction of hardness and toughness of hierarchical materials. Chaoes, Solitons and Fractals 42, 2546-2552.

Carpinteri, A., Puzzi, S., 2009. The fractal-statistical approach to the size-scale effects on material strength and toughness. Probabilistic Engineering Mechanics 24, 75-83. 
Chen, B.Y., Tay, T.E., Baiz, P.M., Pinho, S.T., 2013. Numerical analysis of size effects on open-hole tensile composite laminates. Composites Part A - Applied Science and Manufacturing 47, 52-62.

Chiang, C.R., 2000. Prediction of the fracture toughness of fibrous composites. Journal of Materials Science 35, 3161-3166.

Curtin, W.A., 1991. Theory of mechanical properties of ceramic-matrix composites. Journal of the American Ceramics Society 74, 2837-2845.

Curtin, W.A., 1993. Fiber pull-out and strain localization in ceramic matrix composites. Journal of the Mechanics and Physics of Solids 41, 35-53.

Daniels, H.E., 1945. The statistical theory of the strength of bundles of threads. I. Proceedings of the Royal Society A - Mathematical, Physical and \& Engineering Science 183, 405-435.

Feraboli, P., Peitso, E., Cleveland, T., Sticker, P.B., Halpin, J.C., 2009. Notched behavior of prepreg-based discontinuous carbon fiber/epoxy systems. Composites Part A — Applied Science and Manufacturing 40, 289-299.

Gao, Y.C., Mai, Y.W., Cotterell, B., 1988. Fracture of fiber-reinforced materials. Journal of Applied Mathematics and Physics 39, 550-572.

Harlow, D.G., Phoenix, S.L., 1978a. The chain-of-bundles probability model for the strength of fibrous materials. I: Analysis and conjectures. Journal of Composite Materials 12, 195-214.

Harlow, D.G., Phoenix, S.L., 1978b. The chain-of-bundles probability model for the strength of fibrous materials. II: A numerical study of convergence. Journal of Composite Materials 12, 314-334.

Harper, L.T., Turner, T.A., Warrior, N.A., Rudd, C.D., 2007. Characterisation of random carbon fibre composites from a directed fibre preforming process: The effect of tow filamentisation. Composites Part A - Applied Science and Manufacturing $38,755-770$.

Ilyas, M., Lachaud, F., Espinosa, C., Salaün, M., 2009. Dynamic delamination of aeronautic structural composites by using cohesive finite elements, in: 17th International Conference on Composite Materials, ICCM, Edinburgh, Scotland. 
Jehangir, Z., 2011. Experimental Investigation of the Translaminar Ply Fracture Toughness of Advanced Composites. MEng Project Report. Imperial College London, UK.

Kelly, A., 1970. Interface effects and work of fracture of a fibrous composite. Proceedings of the Royal Society of London Series A - Mathematical and Physical Sciences 319, $95-116$.

Kim, J.K., Baillie, C., Poh, J., Mai, Y.W., 1992. Fracture toughness of CFRP with modified epoxy resin matrices. Composites Science and Technology 42, 283-297.

Kim, J.K., Mai, Y.W., 1991. High strength, high fracture toughness fibre composites with interface control - A review. Composites Science and Technology 41, 333-378.

Laffan, M.J., Pinho, S.T., Robinson, P., Iannucci, L., 2010. Measurement of the in situ ply fracture toughness associated with mode I fibre tensile failure in FRP. Part II: Size and lay-up effects. Composites Science and Technology 70, 614-621.

Laffan, M.J., Pinho, S.T., Robinson, P., McMillan, A.J., 2012. Translaminar fracture toughness testing of composites: A review. Polymer Testing 31, 481-489.

Lim, J.T., Piggott, M.R., Bailey, W.J., 1984. Toughness of fiber composites with controlled matrix shrinkage. SAMPE Quarterly 15, 25-30.

McCarroll, C.A., 2008. Material Characterisation of T300/920. Technical Report. Imperial College London, UK.

van der Meer, F.P., Oliver, C., Sluys, L.J., 2010. Computational analysis of progressive failure in a notched laminate including shear nonlinearity and fiber failure. Composites Science and Technology 70, 692-700.

Pimenta, S., Pinho, S.T., 2012. The effect of recycling on the mechanical response of carbon fibres and their composites. Composite Structures 94, 3669-3684.

Pimenta, S., Pinho, S.T., 2013. Hierarchical scaling law for the strength of composite fibre bundles. Journal of the Mechanics and Physics of Solids 61, 1337-1356.

Pimenta, S., Pinho, S.T., 2014. The influence of micromechanical properties and reinforcement architectures on the mechanical response of recycled composites. Composites Part A - Applied Science and Manufacturing 56, 213-225. 
Pimenta, S., Pinho, S.T., Robinson, P., Wong, K., Pickering, S.J., 2010. Mechanical analysis and toughening mechanisms of a multiphase recycled CFRP. Composites Science and Technology 70, 1713-1725.

Pinho, S., Robinson, P., Iannucci, L., 2006a. Fracture toughness of the tensile and compressive fibre failure modes in laminated composites. Composites Science and Technology 66, 2069-2079.

Pinho, S., Robinson, P., Iannucci, L., 2006b. Physically based failure models and criteria for laminated fibre-reinforced composites with emphasis on fibre kinking. Part II: FE implementation. Composites Part A — Applied Science and Manufacturing 37, 766777.

Teixeira, R., Pinho, S.T., Robinson, P., 2011. Translaminar ply fracture toughness of advanced composites, in: 18th International Conference on Composite Materials, ICCM, Jeju Island, South Korea.

Toray Carbon Fibers America, Inc, 2012. Torayca T300 Data Sheet - Technical Data Sheet No. CFA-001. http://www.toraycfa.com/pdfs/T300DataSheet.pdf.

Weiss, J., 2001. Self-affinity of fracture surfaces and implications on a possible size effect on fracture energy. International Journal of Fracture 109, 365-381.

Wells, J.K., Beaumont, P.W.R., 1982. Fracture energy maps for fibre composites. Journal of Materials Science 17, 397-405.

Wells, J.K., Beaumont, P.W.R., 1985a. Crack-tip energy-absorption processes in fiber composites. Journal of Materials Science 20, 2735-2749.

Wells, J.K., Beaumont, P.W.R., 1985b. Debonding and pull-out processes in fibrous composites. Journal of Materials Science 20, 1275-1284.

Zhang, X., Liu, H.Y., Mai, Y.W., Diao, X.X., 1999. On steady-state fibre pull-out I - The stress field. Composites Science and Technology 59, 2179-2189.

Zhou, L.M., Kim, J.K., Mai, Y.W., 1992. On the single fiber pull-out problem: effect of loading method. Composites Science and Technology 45, 153-160. 